\title{
Neutron electric polarizability from unquenched lattice QCD using the background field approach
}

\author{
M. Engelhardt \\ (LHPC Collaboration) \\ Physics Department, New Mexico State University \\ Las Cruces, NM 88003, USA
}

\begin{abstract}
A calculational scheme for obtaining the electric polarizability of the neutron in lattice QCD with dynamical quarks is developed, using the background field approach. The scheme differs substantially from methods previously used in the quenched approximation, the physical reason being that the QCD ensemble is no longer independent of the external electromagnetic field in the dynamical quark case. One is led to compute (certain integrals over) four-point functions. Particular emphasis is also placed on the physical role of constant external gauge fields on a finite lattice; the presence of these fields complicates the extraction of polarizabilities, since it gives rise to an additional shift of the neutron mass unrelated to polarizability effects. The method is tested on a $S U(3)$ flavor-symmetric ensemble furnished by the MILC Collaboration, corresponding to a pion mass of $m_{\pi}=759 \mathrm{MeV}$. Disconnected diagrams are evaluated using stochastic estimation. A small negative electric polarizability of $\alpha=(-2.0 \pm 0.9) \cdot 10^{-4} \mathrm{fm}^{3}$ is found for the neutron at this rather large pion mass; this result does not seem implausible in view of the qualitative behavior of $\alpha$ as a function of $m_{\pi}$ suggested by Chiral Effective Theory.
\end{abstract}

PACS: 12.38.Gc, 13.40.-f, 13.60.Fz

Keywords: Lattice QCD, hadron structure, polarizability 


\section{Introduction}

An important characteristic of hadrons is their stiffness when subjected to outside forces, which are typically conveyed by external electromagnetic fields. This response is summarized in hadron polarizabilities. Understanding these quantities will contribute to making hadron structure more palpable. Experimentally, polarizabilities are accessible, e.g., via soft Compton scattering; heuristically, in such an experiment, the photon electric and magnetic fields polarize the target hadron, which in turn manifests itself in the Compton scattering amplitude observed. Accordingly, polarizabilities are effects of second order in the external fields.

The aforementioned sensitivity of low-energy Compton scattering to hadron structure can be cast in precise terminology [1], permitting stringent tests of theoretical understanding of that structure. Starting with the leading order in the low-energy expansion, the non-Born (i.e., structure-dependent) part of the scattering amplitude is determined by the static dipole electric and magnetic polarizabilities $\alpha$ and $\beta$. These are given by the hadron mass shift in the presence of external static electric and magnetic fields, specifically the part of the mass shift which depends quadratically on those fields, in accordance with a (spin-independent) effective dipole interaction Hamiltonian

$$
H_{e f f}^{(2)}=-\frac{1}{2}\left(\alpha E^{2}+\beta B^{2}\right) .
$$

The present investigation focuses on the electric polarizability $\alpha$ of the neutron.

Lattice hadron polarizability calculations have hitherto been carried out only in the quenched approximation [2-9]. The reason for this lies in the fact that, in the case of polarizabilities, the complication implied by going from a quenched to an unquenched calculation involves more than just the usual vastly increased effort required to generate a dynamical quark ensemble. In addition, a quenched calculation is simpler due to the gauge ensemble being independent of the external electromagnetic field; after all, the only way the external field can influence the gauge ensemble is through the quarks, whose backreaction on the gauge fields is precisely truncated in a quenched calculation. This is no longer true in the dynamical quark case.

This physical difference manifests itself formally in the fact that substantially different computational schemes have to be used in the dynamical quark case as compared to the quenched case. In the quenched case, one can simply generate gauge configurations in the absence of the external electromagnetic field and introduce the latter a posteriori by an appropriate modification of the link variables in those configurations. The requisite hadron two-point functions are then evaluated directly using the modified gauge configurations. By contrast, in a fully dynamical calculation, as discussed in more detail below, one in principle would need to generate the gauge ensemble anew for each external field considered. The prohibitive cost of such a scheme can be mitigated to some extent by expanding in the external field, leading, in effect, to the calculation of (certain 
space-time integrals over) four-point functions. In general, these include disconnected contributions. However, even resorting to such a four-point function method leads to a substantially more expensive calculation than one is confronted with in the quenched case; an early exploratory study of four-point function methods [3] (using a quenched ensemble) highlights this point 1 .

The most of this situation has been made hitherto in a series of investigations [2,5-9] taking full advantage of the simplifications offered by the quenched approximation. An initial study of the electric polarizability of neutral hadrons [2] using staggered fermions yielded results both for the neutral pion and the neutron. This was later extended to include all neutral members of the baryon octet, as well as the baryon decuplet and the vector meson octet, in investigations using both Wilson and clover fermions [5,7]; these studies surveyed a range of pion masses down to about $500 \mathrm{MeV}$. Also the magnetic polarizability of a wide array of hadrons was investigated using the same range of pion masses and fermion actions [6, 8,9]; these studies included also charged hadrons, in particular the entire baryon octet and decuplet as well as selected pseudoscalar and vector mesons.

The present work extends the aforementioned work in two main aspects:

- Use of a dynamical quark ensemble: As discussed above, dynamical quark calculations of polarizabilities were rendered intractable in the past by the associated computational cost. Recent increases in available computing resources are making quantitative four-point function calculations, appropriate for dynamical ensembles, feasible. This investigation presents the first result for the electric polarizability of the neutron in a dynamical quark ensemble, albeit obtained at a still rather heavy pion mass of $759 \mathrm{MeV}$.

- Recognition that, on a finite spatial volume, a constant gauge field is not a pure gauge, but has physical consequences which must be disentangled from polarizability effects.

Some elaboration on the latter issue, which is also relevant in the quenched approximation, is useful at this point. As noted further above, hadron polarizabilities can be probed via the mass shift in the presence of external electromagnetic fields. The hitherto preferred method $[2,5,7]$ of introducing a constant electric field in, say, the 3-direction is to represent it by a non-vanishing 3-component of the gauge field,

$$
A_{3}=E\left(t-t_{0}\right)
$$

This choice has the advantage that jumps in the gauge field at the lattice boundaries (inducing spurious localized electric fields there) occur only in the temporal direction. In

\footnotetext{
${ }^{1}$ The four-point functions considered in [3] are different from the ones which are calculated in the present work, since different theoretical approaches are used. However, the computational complexity resulting from the two approaches is similar.
} 
this case, hadron two-point functions evaluated in the bulk of the lattice are insensitive to the jumps, which only occur far in the past or the future from the point of view of the measurement.

However, there is an ambiguity in the prescription (2), namely, at which time $t_{0}$ one chooses to begin counting time. Different choices of $t_{0}$ correspond to different constant shifts of $A_{3}$. Working in a spatially infinite setting, this ambiguity would be inconsequential, since constant gauge fields are then pure gauges. However, on a finite space, the spatial boundary conditions (which in the following will be taken to be periodic) restrict the available gauge transformations and only allow for discrete shifts of the gauge fields. As a simple example, consider a charged particle in a constant field on a circle of length $L$ described by the Hamiltonian $H=\left(-i \partial_{x}+A\right)^{2}$. Its energy eigenvalues are $E_{n}=(2 \pi n / L+A)^{2}$, where $n$ can be any integer. The ground state energy therefore is $E_{0}=A^{2}$ as long as $A \in[-\pi / L, \pi / L]$, and $E_{0}$ is periodic in $A$ with period $2 \pi / L$, reflecting the residual discrete gauge invariance. The spectrum explicitly depends on $A$ and, compared with the case $A=0$, the ground state energy can deviate by as much as $\Delta E_{0}=\pi^{2} / L^{2}$. Thus, while this is ultimately nothing but a finite size effect, it vanishes rather slowly (only as a power of $L$ ) as the spatial volume is increased. It is a priori unclear how difficult it is in practice to deal with this effect by using different lattice sizes. Within the present investigation, that avenue is closed at any rate, since the dynamical quark ensemble which will be used is only available at one spatial volume. Instead, measurements at several different $t_{0}$ in (2) will be used in order to treat this effect.

Another aspect of the same issue is that the Hamiltonian in the presence of the field (21) is not time-independent. Physics at two widely separated times differ precisely by a shift in the external gauge field $A_{3}$. If the electric field $E$ is very small, the strong dynamics can instantaneously adjust to the change in the external field as time passes; one will observe an adiabatic change in the physical spectrum. The hadronic two-point function will generally not fall off as a simple exponential in Euclidean time, even for large such times. Both the hadron's energy as well as its wave function will contain time dependences. This is reminiscent of the behavior induced by the acceleration of charged hadrons in the electric field [10]. That particular effect is negligible for sufficiently heavy hadrons, such as nucleons. By contrast, the constant gauge field effect discussed here is one of the dominant effects, and care needs to be taken to disentangle it from the nucleon polarizability.

A way to avoid the time dependence discussed above is to instead use a gauge field representation of the type

$$
A_{0}=-E x_{3}
$$

inducing the same external electric field as (2); indeed, in the present work, also this case will be investigated. However, it should be noted that this choice also has disadvantages which, on balance, are no less problematic than the time dependence engendered by (2). 
Namely, the advantage of time-independence using (3) is offset by the fact that spatial translational invariance is lost; the representation (3) conflicts with spatial periodicity and the periodic boundary conditions enforce a spike in the electric field resulting from the jump in $A_{0}$ as one crosses the boundary of the lattice in the 3-direction 2 . In effect, the neutron thus propagates in a spatially varying potential and its energy contains, e.g., contributions from quantum mechanical zero-point motion in that potential. Also the neutron's internal wave function is distorted by the electric field spikes. As a consequence, it is not straightforward to isolate the polarizability from the full measured mass shift.

\section{Measurement method}

\subsection{Neutron two-point function}

The objective of the present investigation is to extract the neutron mass from the neutron two-point function in the presence of an external electric field. The neutron two-point function is the correlator

$$
\left\langle N_{\alpha^{\prime}}\left(x^{\prime}\right) \bar{N}_{\alpha}(x)\right\rangle=\frac{1}{Z} \int[D U][D \bar{\psi}][D \psi] \exp (-S[\psi, \bar{\psi}, U]) N_{\alpha^{\prime}}\left(x^{\prime}\right) \bar{N}_{\alpha}(x),
$$

with the lattice discretization of the functional integral to be specified below. Both the action $S$ and the (smeared) neutron fields $N, \bar{N}$ in general depend on the external electromagnetic field $A_{\mu}$. At face value, this would imply that one needs to generate lattice ensembles using an action modified by the external field in order to evaluate (4). That would clearly be prohibitively expensive using dynamical quarks. However, decomposing the action as

$$
S=S_{0}+S_{E}
$$

where $S_{0}$ is the action in the case of vanishing external field, one can rewrite (4) as

$$
\left\langle N_{\alpha^{\prime}}\left(x^{\prime}\right) \bar{N}_{\alpha}(x)\right\rangle=\frac{\left\langle e^{-S_{E}} N_{\alpha^{\prime}}\left(x^{\prime}\right) \bar{N}_{\alpha}(x)\right\rangle_{0}}{\left\langle e^{-S_{E}}\right\rangle_{0}},
$$

with $\langle\ldots\rangle_{0}$ denoting the average in the absence of the external field,

$$
\langle O\rangle_{0}=\frac{1}{Z_{0}} \int[D U][D \bar{\psi}][D \psi] \exp \left(-S_{0}\right) O
$$

\footnotetext{
${ }^{2}$ Note that, in the lattice formulation, there exist discrete choices of $E$ which mitigate this problem, namely, integer multiples of $2 \pi / a L$, where $L$ is the extent of the lattice in the relevant direction and $a$ denotes the lattice spacing. However, this argument relies on the compactness of the gauge link variables and, on realistic lattices, corresponds to strong electric fields. By contrast, hadron electric polarizabilities are given specifically by the term quadratic in $E$ of a Taylor expansion of their mass. To isolate this term, it is necessary to vary $E$ over a denser set of values than provided by the aforementioned discrete choices, for which the Taylor expansion will generally not converge well on lattices of a practical size.
} 
While this reduces the problem to integrations over the lattice ensemble in the absence of the external field, expectation values such as the ones in (6) generally suffer from severe overlap problems. The crucial step which renders the problem somewhat more tractable results from the fact that it is sufficient to know the quadratic term in the Taylor expansion of (6) with respect to the external field in order to extract the neutron polarizability. Then, one can expand

$$
\exp \left(-S_{E}\right)=1-S_{E}+S_{E}^{2} / 2+\ldots
$$

and the evaluation of (6) reduces to the calculation of certain space-time integrals over four-point functions, as will be discussed in detail below. Before proceeding to describe this perturbative expansion, it is now possible to specify how the functional integration in (17) will be carried out. As usual, decomposing $S_{0}$ into its pure gauge and fermion parts,

$$
S_{0}=S_{G}+S_{F},
$$

the integration is cast in terms of an average over an ensemble of gauge fields $U$,

$$
\langle O\rangle_{0}=\frac{1}{Z_{0}} \int[D U] \exp \left(-S_{G, e f f}\right)\langle O\rangle_{U},
$$

governed by the action $S_{G, e f f}$ which includes the effects of both the pure gauge term as well as the determinant of the Dirac operator from $S_{F}$. Adopting $2+1$ flavor Asqtad quark fields to evaluate the determinant, one can utilize the corresponding dynamical quark ensembles made available by the MILC Collaboration [11-13]. The numerical results reported in the present work were obtained using 99 configurations from the $S U(3)$ flavor-symmetric ensemble with quark masses given by $a m_{s}=a m_{l}=0.05$, where the lattice spacing $a=0.124 \mathrm{fm}$ is determined by heavy quark spectroscopy [14]. Computation at such a relatively large quark mass is comparatively inexpensive and serves primarily to validate the concepts developed in this work as well as giving a first indication of the feasibility of a broader calculational effort within the framework advanced here.

The aforementioned configurations were originally generated on $20^{3} \times 64$ lattices. In the present work, these lattices were chopped in half in the time direction, i.e., measurements were carried out on $20^{3} \times 32$ lattices containing the first 32 time slices of the original $20^{3} \times 64$ lattices. Correspondingly, quark propagators determining the quantities $\langle O\rangle_{U}$, cf. (11), were evaluated using Dirichlet boundary conditions at the temporal edges of the chopped lattices. The lattices were furthermore HYP-smeared [15] to reduce the effect of dislocations.

For a given gauge configuration $U$, the expectation value

$$
\langle O\rangle_{U}=\frac{\int[D \bar{\psi}][D \psi] \exp \left(-S_{F}\right) O}{\int[D \bar{\psi}][D \psi] \exp \left(-S_{F}\right)}
$$


needs to be evaluated. At this point, a hybrid approach is adopted $[16,17]$ : While the gauge ensemble used in the average (10) is generated using Asqtad quarks, (11) is evaluated using domain-wall quarks $[18,19]$. The reason for this choice lies in the longerterm goal of extending the present investigation to light quark masses at which a chirally well-behaved quark discretization becomes important. Thus, the fields $\psi, \bar{\psi}$ in (11) are taken to be five-dimensional, $\psi(x, s)$ and $\bar{\psi}(x, s)$, where $x$ labels four-dimensional spacetime and $s$ labels the fifth coordinate. The latter is subdivided into $L_{s}=16$ spacings, $s \in\left\{0, \ldots, L_{s}-1\right\}$, and $\psi(x, s) \equiv 0$ outside that range (i.e., derivatives in the fifth direction have hard boundaries). This value of $L_{s}$ is sufficient to keep the residual mass $m_{\text {res }}$ which characterizes explicit chiral symmetry breaking by the domain-wall fermion discretization suppressed by more than an order of magnitude compared to the quark mass discussed further below [16,17]. The boundaries $s=0$ and $s=L_{s}-1$ provide domain walls which support quasi-four-dimensional light physical quark modes; lefthanded modes are bound to $s=0$ and right-handed modes to $s=L_{s}-1$. It is useful to define corresponding four-dimensional projected quark fields

$$
\begin{aligned}
\Psi(x) & =\frac{1-\gamma_{5}}{2} \psi(x, 0)+\frac{1+\gamma_{5}}{2} \psi\left(x, L_{s}-1\right) \\
\bar{\Psi}(x) & =\bar{\psi}(x, 0) \frac{1+\gamma_{5}}{2}+\bar{\psi}\left(x, L_{s}-1\right) \frac{1-\gamma_{5}}{2}
\end{aligned}
$$

In terms of the above fields, the action $S_{F}$ in (11) reads

$$
\begin{aligned}
S_{F}[\psi, \bar{\psi}, U]= & -\sum_{x, s} \sum_{\mu} \bar{\psi}(x, s)\left(\frac{1-\gamma_{\mu}}{2}\left[U_{\mu}(x) \psi\left(x+e_{\mu}, s\right)-\psi(x, s)\right]\right. \\
& \left.-\frac{1+\gamma_{\mu}}{2}\left[-U_{\mu}^{\dagger}\left(x-e_{\mu}\right) \psi\left(x-e_{\mu}, s\right)+\psi(x, s)\right]\right) \\
& -\sum_{x, s} \bar{\psi}(x, s) M_{5} \psi(x, s)+\sum_{x} \bar{\Psi}(x) m_{f} \Psi(x)
\end{aligned}
$$

where $\mu$ runs over all five dimensions and $U_{5} \equiv 1$. Note that the fermion fields also carry a flavor index; in the final term, i.e., the quark mass term, which is constructed directly in terms of the projected four-dimensional quark fields $\Psi$ and $\bar{\Psi}, m_{f}$ in general represents a (diagonal) matrix in flavor space. In the $S U(3)$ flavor-symmetric case studied here, $m_{f}$ is given by one single number.

Using spectral flow analyses, the five-dimensional mass parameter $M_{5}$ in (14) was chosen to take the value $M_{5}=1.7$ in order to optimize the chiral properties $[16,17]$. Finally, the quark mass was adjusted such as to match the pion mass obtained in the present hybrid approach to the lightest pion mass extracted from a pure Asqtad calculation [14]; this yields $[16,17]$ the choice $a m_{f}=0.081$.

The domain wall fermion action (14) also determines the interaction between the quarks and the external electric field. The additional electromagnetic gauge field $A_{\mu}$ 
generating the external electric field modifies the gauge link variables,

$$
U_{\mu}(x) \longrightarrow \exp \left(i a q_{f} A_{\mu}(x)\right) U_{\mu}(x)
$$

where $a$ denotes the lattice spacing; note that the fractional electric charge $q_{f}$ varies according to flavor. Note also that the particular forms of $A_{\mu}$ used in this work, cf. (2), (3), are all such that $A_{\mu}$ is constant in the $\mu$-direction; hence the simple form (15) for the exponentiated integral along the link. Inserting the modified link variables (15) into the domain wall fermion action (14) and separating off the part which remains for vanishing external field, $A_{\mu}=0$, yields the five-dimensional interaction

$$
\begin{aligned}
S_{E, 5 d}=-\sum_{x, s} \sum_{\mu} \bar{\psi}(x, s) & \left(\frac{1-\gamma_{\mu}}{2}\left(e^{i a q_{f} A_{\mu}(x)}-1\right) U_{\mu}(x) \psi\left(x+e_{\mu}, s\right)\right. \\
+ & \left.\frac{1+\gamma_{\mu}}{2}\left(e^{-i a q_{f} A_{\mu}\left(x-e_{\mu}\right)}-1\right) U_{\mu}^{\dagger}\left(x-e_{\mu}\right) \psi\left(x-e_{\mu}, s\right)\right)
\end{aligned}
$$

generating a vertex which couples the five-dimensional domain wall fermion fields $\psi, \bar{\psi}$ to the external field.

To arrive at a practicable computational scheme, in the calculations presented further below, the external gauge field $A_{\mu}$ is not coupled directly to the five-dimensional fields according to (16), but instead to the corresponding four-dimensional projected quark fields $\Psi, \bar{\Psi}$. Accordingly, a renormalization factor $z_{V}$ must be included with the fourdimensional coupling to compensate for the effect of the projection of the quark fields. Thus, the modified interaction vertex used in practice is

$$
\begin{aligned}
S_{E}=-z_{V} \sum_{x} \sum_{\mu} \bar{\Psi} & (x)\left(\frac{1-\gamma_{\mu}}{2}\left(e^{i a q_{f} A_{\mu}(x)}-1\right) U_{\mu}(x) \Psi\left(x+e_{\mu}\right)\right. \\
& \left.+\frac{1+\gamma_{\mu}}{2}\left(e^{-i a q_{f} A_{\mu}\left(x-e_{\mu}\right)}-1\right) U_{\mu}^{\dagger}\left(x-e_{\mu}\right) \Psi\left(x-e_{\mu}\right)\right) .
\end{aligned}
$$

The renormalization factor $z_{V}$ will be determined in section 4.1. The reason for the adoption of the modified interaction (17) lies in the practical expense of storing full fivedimensional propagators as opposed to ones which have been projected down to four dimensions at source and sink. This modus operandi constitutes a compromise which certainly should be revisited as storage constraints change. Using full five-dimensional propagators and coupling the conserved five-dimensional current to the external electromagnetic field directly via (16) would be the most consistent treatment, and would eliminate the need for renormalization of the interaction vertex.

Finally, it is necessary to specify the neutron sources and $\operatorname{sinks} \bar{N}, N$ in (4):

$$
\begin{aligned}
& N_{\alpha}(x)=\delta_{\alpha \beta}\left(C \gamma_{5}\right)_{\gamma \delta} \epsilon_{b c d} Q_{b \beta}^{(d)}(x) Q_{c \gamma}^{(d)}(x) Q_{d \delta}^{(u)}(x) \\
& \bar{N}_{\alpha}(x)=\bar{Q}_{d \delta}^{(u)}(x) \bar{Q}_{c \gamma}^{(d)}(x) \bar{Q}_{b \beta}^{(d)}(x) \epsilon_{b c d} \delta_{\alpha \beta}\left(C \gamma_{5}\right)_{\gamma \delta}
\end{aligned}
$$


where $C$ denotes the charge conjugation operator and $Q$ is a Wuppertal-smeared [20] quark field (with the superscript denoting flavor), constructed iteratively as (where the superscript now momentarily labels iterations):

$$
Q^{(i)}(x)=(1-6 \sigma) Q^{(i-1)}(x)+\sigma \sum_{\mu= \pm 1}^{ \pm 3} U_{\mu}^{\dagger}\left(x-e_{\mu}\right) Q^{(i-1)}\left(x-e_{\mu}\right) .
$$

Here, $\sigma$ and the number of iterations $i_{\text {max }}$ are free parameters, chosen such as to generate a good overlap between the neutron source and the true neutron ground state [21]. The iteration starts at $Q^{(0)} \equiv \Psi$ and ends at $Q^{\left(i_{\max }\right)} \equiv Q$. The sum over directions $\mu$ in (20) runs only over the three spatial dimensions, but includes terms associated with both positive and negative displacements in each dimension (i.e., $e_{-\mu}=-e_{\mu}$, $\left.U_{-\mu}^{\dagger}\left(x-e_{-\mu}\right)=U_{\mu}(x)\right)$. Note that smearing constitutes a linear operation on the quark fields, i.e., there exists a matrix $P$ such that

$$
Q(x)=P(x, y) \Psi(y), \quad \bar{Q}(x)=\bar{\Psi}(y) P^{\dagger}(y, x) .
$$

$P$ is proportional to the unit matrix in the Dirac indices, but not in the space-time and color indices, nor in the flavor indices once the external electric field is introduced via the substitution (15). Note, thus, that the presence of the external electric field can influence the smearing if one insists on manifest invariance of the neutron sources and sinks with respect to gauge transformations of the external field. However, it is not imperative to preserve such manifest invariance; not doing so merely corresponds to evaluating (gaugeinvariant) physical observables in a particular gauge. In the treatment to follow, the most general case will be considered, i.e., the perturbative expansion discussed below will include the diagrams resulting from expanding the source and sink fields in the external field. This will make it possible to separately assess the influence of such terms. Ultimately, unambiguous extraction of the neutron electric polarizability will be seen to necessitate discarding such diagrams, and thus foregoing manifest invariance of the neutron sources and sinks with respect to gauge transformations of the external field; nevertheless, it will be verified that the effect of including additional smearing diagram contributions on the final result for the polarizability is negligible, thus rendering this issue moot in any case.

\subsection{Perturbative expansion}

Having defined all of the objects entering the neutron two-point function (4), one can proceed to extract the quadratic term of its Taylor expansion with respect to the external field $A_{\mu}$. Both the interaction $S_{E}$ and the smeared neutron sources $N$ and $\bar{N}$ in general contain a dependence on $A_{\mu}$. Expanding (17), one obtains two relevant vertices,

$$
S_{E}=S_{E, 1}+S_{E, 2}+O\left(A_{\mu}^{3}\right)
$$


which can be written as bilinear forms,

$$
S_{E, i}=\bar{\Psi} M_{i} \Psi
$$

with

$$
\begin{aligned}
& M_{1}(x, y)=-i a z_{V} q_{f} \sum_{\mu}( \frac{1-\gamma_{\mu}}{2} A_{\mu}(x) U_{\mu}(x) \delta\left(x+e_{\mu}, y\right) \\
&\left.-\frac{1+\gamma_{\mu}}{2} A_{\mu}\left(x-e_{\mu}\right) U_{\mu}^{\dagger}\left(x-e_{\mu}\right) \delta\left(x-e_{\mu}, y\right)\right) \\
& M_{2}(x, y)=\frac{a^{2}}{2} z_{V} q_{f}^{2} \sum_{\mu}\left(\frac{1-\gamma_{\mu}}{2} A_{\mu}^{2}(x) U_{\mu}(x) \delta\left(x+e_{\mu}, y\right)\right. \\
&\left.+\frac{1+\gamma_{\mu}}{2} A_{\mu}^{2}\left(x-e_{\mu}\right) U_{\mu}^{\dagger}\left(x-e_{\mu}\right) \delta\left(x-e_{\mu}, y\right)\right) .
\end{aligned}
$$

Thus, $M_{1}$ and $M_{2}$ are matrices in the space-time, color, Dirac and flavor indices, summation over which is implied in (23).

On the other hand, also the smeared fields defined by (20) need to be expanded in the external field,

$$
Q^{(i)}=Q_{0}^{(i)}+Q_{1}^{(i)}+Q_{2}^{(i)}+O\left(A_{\mu}^{3}\right)
$$

(where the subscript denotes the order in the external field). Modifying the link variables in (20) according to (15) and expanding in $A_{\mu}$, one has an iterative construction of the smeared fields separated order by order in the external gauge field,

$$
\begin{gathered}
Q_{0}^{(i)}(x)=(1-6 \sigma) Q_{0}^{(i-1)}(x)+\sigma \sum_{\mu= \pm 1}^{ \pm 3} U_{\mu}^{\dagger}\left(x-e_{\mu}\right) Q_{0}^{(i-1)}\left(x-e_{\mu}\right) \\
Q_{1}^{(i)}(x)=(1-6 \sigma) Q_{1}^{(i-1)}(x)+\sigma \sum_{\mu= \pm 1}^{ \pm 3} U_{\mu}^{\dagger}\left(x-e_{\mu}\right)\left(Q_{1}^{(i-1)}\left(x-e_{\mu}\right)\right. \\
Q_{2}^{(i)}(x)=(1-6 \sigma) Q_{2}^{(i-1)}(x)+\sigma \sum_{\mu= \pm 1}^{ \pm 3} U_{\mu}^{\dagger}\left(x-e_{\mu}\right)\left(Q_{2}^{(i-1)}\left(x-e_{\mu}\right)\right. \\
\left.-i a q_{f} A_{\mu}\left(x-e_{\mu}\right) Q_{0}^{(i-1)}\left(x-e_{\mu}\right)\right) \\
\left.-\frac{a^{2} q_{f}^{2}}{2} A_{\mu}^{2}\left(x-e_{\mu}\right) Q_{0}^{(i-1)}\left(x-e_{\mu}\right)\right)
\end{gathered}
$$

Equivalently, the smearing matrix $P$ in (21) can be written in expanded fashion,

$$
P=P_{0}+P_{1}+P_{2}+O\left(A_{\mu}^{3}\right)
$$

(the original quark field $\Psi$ is of course of zeroth order in $A_{\mu}$ ). 
Returning to the neutron two-point function, expanding (므) in powers of $S_{E}$, inserting (22) and discarding terms which contribute only at higher than quadratic order in the external field yields

$$
\begin{aligned}
\left\langle N_{\alpha^{\prime}}\left(x^{\prime}\right) \bar{N}_{\alpha}(x)\right\rangle=\langle(1- & \left.\left.S_{E}+S_{E}^{2} / 2\right) N_{\alpha^{\prime}}\left(x^{\prime}\right) \bar{N}_{\alpha}(x)\right\rangle_{0}\left(1+\left\langle S_{E}-S_{E}^{2} / 2\right\rangle_{0}+\left\langle S_{E}\right\rangle_{0}^{2}\right) \\
= & \left\langle N_{\alpha^{\prime}}\left(x^{\prime}\right) \bar{N}_{\alpha}(x)\right\rangle_{0} \\
& -\left\langle\left(S_{E, 1}+S_{E, 2}-S_{E, 1}^{2} / 2\right) N_{\alpha^{\prime}}\left(x^{\prime}\right) \bar{N}_{\alpha}(x)\right\rangle_{0} \\
& +\left\langle S_{E, 1}+S_{E, 2}-S_{E, 1}^{2} / 2\right\rangle_{0}\left\langle N_{\alpha^{\prime}}\left(x^{\prime}\right) \bar{N}_{\alpha}(x)\right\rangle_{0} \\
& -\left\langle S_{E, 1}\right\rangle_{0}\left\langle S_{E, 1} N_{\alpha^{\prime}}\left(x^{\prime}\right) \bar{N}_{\alpha}(x)\right\rangle_{0} \\
& +\left\langle S_{E, 1}\right\rangle_{0}^{2}\left\langle N_{\alpha^{\prime}}\left(x^{\prime}\right) \bar{N}_{\alpha}(x)\right\rangle_{0}
\end{aligned}
$$

As usual, the denominator in the original expression (66) has the effect of subtracting disconnected (in the statistical sense) pieces. Furthermore, inserting the more specific forms (18), (19), (21) and (23), one arrives at (the superscripts of the smearing matrices $P$ and the quark fields $\Psi$ denoting a fixed flavor):

$$
\begin{aligned}
& \left\langle N_{\alpha^{\prime}}\left(x^{\prime}\right) \bar{N}_{\alpha}(x)\right\rangle=\delta_{\alpha^{\prime} \beta^{\prime}}\left(C \gamma_{5}\right)_{\gamma^{\prime} \delta^{\prime}} \epsilon_{b^{\prime} c^{\prime} d^{\prime}} \epsilon_{b c d} \delta_{\alpha \beta}\left(C \gamma_{5}\right)_{\gamma \delta} \\
& \times P_{b^{\prime} k^{\prime}}^{(d)}\left(x^{\prime}, u^{\prime}\right) P_{c^{\prime} l^{\prime}}^{(d)}\left(x^{\prime}, v^{\prime}\right) P_{d^{\prime} m^{\prime}}^{(u)}\left(x^{\prime}, w^{\prime}\right) P_{m d}^{\dagger(u)}(w, x) P_{l c}^{\dagger(d)}(v, x) P_{k b}^{\dagger(d)}(u, x) \\
& \times\left[-\left\langle\Psi_{k^{\prime} \beta^{\prime}}^{(d)}\left(u^{\prime}\right) \Psi_{l^{\prime} \gamma^{\prime}}^{(d)}\left(v^{\prime}\right) \Psi_{m^{\prime} \delta^{\prime}}^{(u)}\left(w^{\prime}\right) \bar{\Psi}_{m \delta}^{(u)}(w) \bar{\Psi}_{l \gamma}^{(d)}(v) \bar{\Psi}_{k \beta}^{(d)}(u)\right.\right. \\
& \left.\times\left(\left(\bar{\Psi} M_{1} \Psi\right)+\left(\bar{\Psi} M_{2} \Psi\right)-\frac{1}{2}\left(\bar{\Psi} M_{1} \Psi\right)\left(\bar{\Psi} M_{1} \Psi\right)\right)\right\rangle_{0} \\
& -\left\langle\Psi_{k^{\prime} \beta^{\prime}}^{(d)}\left(u^{\prime}\right) \Psi_{l^{\prime} \gamma^{\prime}}^{(d)}\left(v^{\prime}\right) \Psi_{m^{\prime} \delta^{\prime}}^{(u)}\left(w^{\prime}\right) \bar{\Psi}_{m \delta}^{(u)}(w) \bar{\Psi}_{l \gamma}^{(d)}(v) \bar{\Psi}_{k \beta}^{(d)}(u)\left(\bar{\Psi} M_{1} \Psi\right)\right\rangle_{0} \\
& \times\left\langle\left(\bar{\Psi} M_{1} \Psi\right)\right\rangle_{0} \\
& +\left\langle\Psi_{k^{\prime} \beta^{\prime}}^{(d)}\left(u^{\prime}\right) \Psi_{l^{\prime} \gamma^{\prime}}^{(d)}\left(v^{\prime}\right) \Psi_{m^{\prime} \delta^{\prime}}^{(u)}\left(w^{\prime}\right) \bar{\Psi}_{m \delta}^{(u)}(w) \bar{\Psi}_{l \gamma}^{(d)}(v) \bar{\Psi}_{k \beta}^{(d)}(u)\right\rangle_{0} \\
& \times\left(1+\left\langle\left(\bar{\Psi} M_{1} \Psi\right)+\left(\bar{\Psi} M_{2} \Psi\right)-\frac{1}{2}\left(\bar{\Psi} M_{1} \Psi\right)\left(\bar{\Psi} M_{1} \Psi\right)\right\rangle_{0}\right. \\
& \left.\left.+\left\langle\left(\bar{\Psi} M_{1} \Psi\right)\right\rangle_{0}^{2}\right)\right]
\end{aligned}
$$

Applying Wick's theorem (i.e., evaluating the $\langle\ldots\rangle_{U}$ averages over the quark fields, cf. (10),(11)), and retaining only contributions quadratic in the external gauge field, one finally arrives at a diagrammatic representation, depicted in Fig. 1, for the desired quantity, namely, the quadratic term in the Taylor expansion of the neutron two-point function with respect to the external field. The diagrams in Fig. 1 are to be read as follows:

a. Solid lines are point-to-point quark propagators $K_{\gamma^{\prime} \gamma}^{(f) c^{\prime} c}\left(x^{\prime}, x\right)=\left\langle\Psi_{c^{\prime} \gamma^{\prime}}^{(f)}\left(x^{\prime}\right) \bar{\Psi}_{c \gamma}^{(f)}(x)\right\rangle_{U}$. Note that these are propagators between four-dimensional sources and sinks, i.e., an 

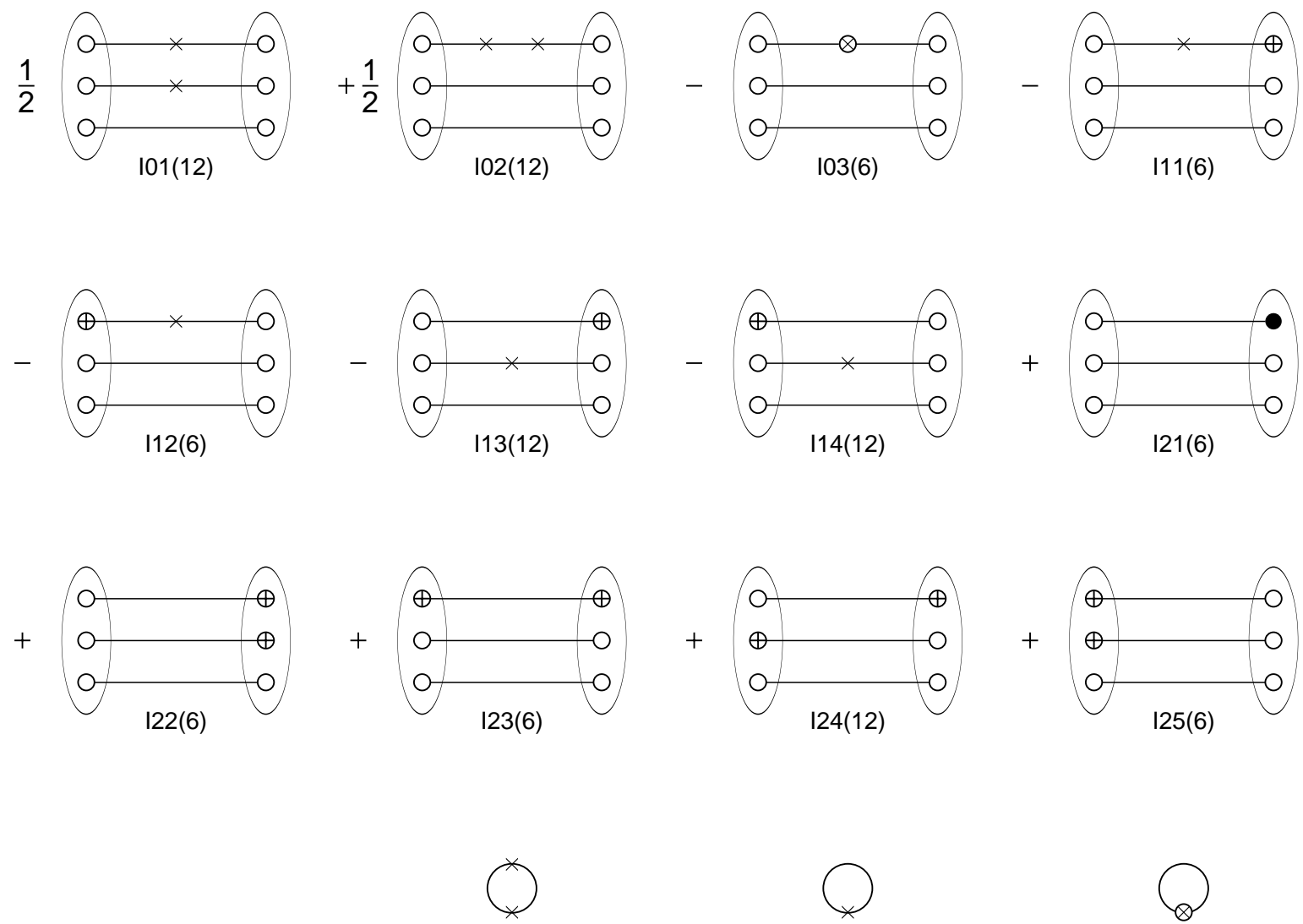

0
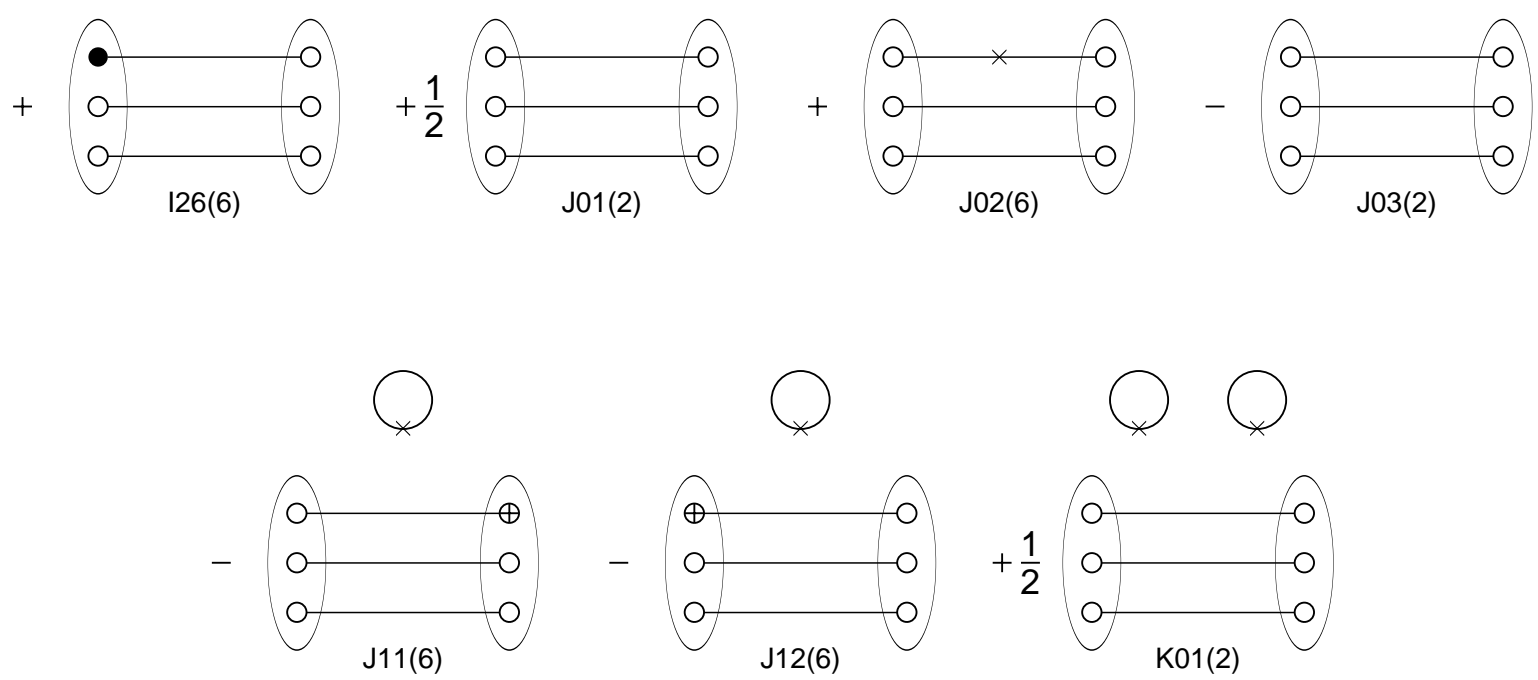

Figure 1: Contributions to the neutron two-point function quadratic in the external gauge field. The nomenclature is explained in detail in the main text. 
initially four-dimensional source is propagated in five dimensions using the domain wall quark action (14) and finally projected back to four-dimensional space-time according to the correspondence (13). Of the three propagator chains connecting neutron source and sink, two are associated with flavor down, $f=d$, and one with flavor up, $f=u$. The quark loops imply a sum over all three flavors.

b. $\bigcirc$ denotes quark source or sink smearing of zeroth order in the external field, i.e. $P_{0}$ in the decomposition (30). Similarly, $\oplus$ corresponds to $P_{1}$ and 0 corresponds to $P_{2}$.

c. $\times$ denotes a vertex insertion linear in the external field, i.e., multiplication by $M_{1}$, cf. (24). Similarly, $\otimes$ corresponds to $M_{2}$, cf. (25).

d. At the neutron source and sink, symbolized by the ovals, color and Dirac indices must be contracted in accordance with the first line of the right-hand side of (32).

e. Each diagram summarizes several elementary terms in the Wick expansion of (32). For every contribution in which the quark lines run literally as shown, there is a corresponding contribution in which the two down quark lines connecting neutron source and sink cross (i.e., the sinks are exchanged). The latter contribution receives an additional minus sign from the exchange. Furthermore, for each diagram, there are several ways of distributing vertices and smearings over the quark sources, sinks and propagators, only one of which is shown in each case. Note that the combinatorics are different for vertices and smearings. On the one hand, there are six ways of distributing two $X$ vertices such that they reside on different quark lines connecting neutron source and sink (i.e., exchanging two such vertices amounts to a new contribution $\left.{ }^{3}\right)$; on the other hand, there are only three ways of distributing two $\bigoplus$ sink smearings in the neutron sink (since the product $\left(P_{0}+P_{1}+P_{2}\right)^{3}$ contains only three terms consisting of two factors $P_{1}$ and one factor $P_{0}$ ), and analogously for the neutron source. Note that the labeling of the diagrams reflects these multiplicities; in each label, the integer inside the parentheses denotes the number of individual contributions from the Wick expansion of (32) summarized by the diagram.

f. As usual, each quark loop implies an additional minus sign. In order to keep with standard nomenclature, these signs were not absorbed into the prefactors, but must be included separately when evaluating the diagrams.

g. Each contribution finally must be averaged over the gauge ensemble, where, as already remarked after eq. (31), statistically disconnected parts are subtracted.

\footnotetext{
${ }^{3}$ To be completely precise, this only applies when both vertices reside on quark lines connecting neutron source and sink; on the other hand, in the diagrams labeled $J 01(2), J 02(6)$ and $K 01(2)$, no additional contributions stemming from exchange of the vertices are implied. Any such duplications which may arise from the Wick expansion of (32) are already taken into account through the prefactor of the diagram (such a duplication actually only occurs in the case of J02(6), the statistically connected part of which, cf. item g., originally enters with a prefactor of $1 / 2$ ).
} 
Thus, denoting the gauge ensemble average as

$$
\langle O\rangle_{G}=\frac{1}{Z_{0}} \int[D U] \exp \left(-S_{G, e f f}\right) O
$$

diagram $J 01(2)$ is to be evaluated as

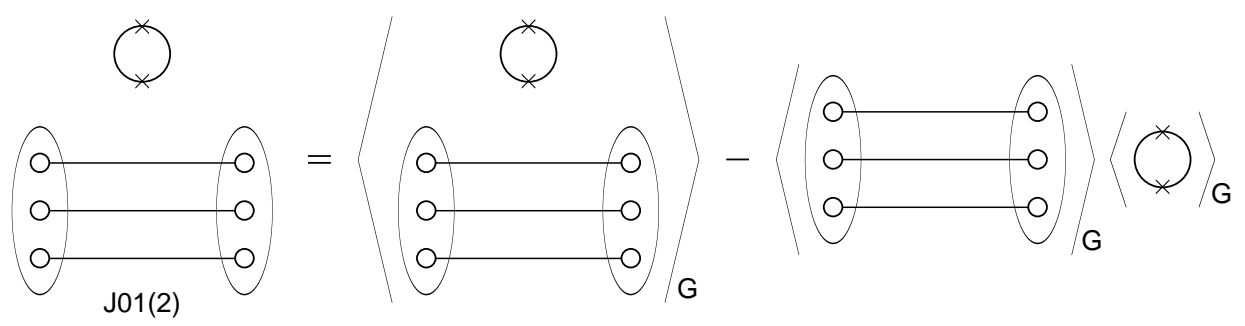

(where of course only items a.-f. apply to the objects inside the averages). Diagrams $J 02(6), J 03(2), J 11(6)$ and $J 12(6)$ are treated analogously. The more complicated case $K 01(2)$ is evaluated as

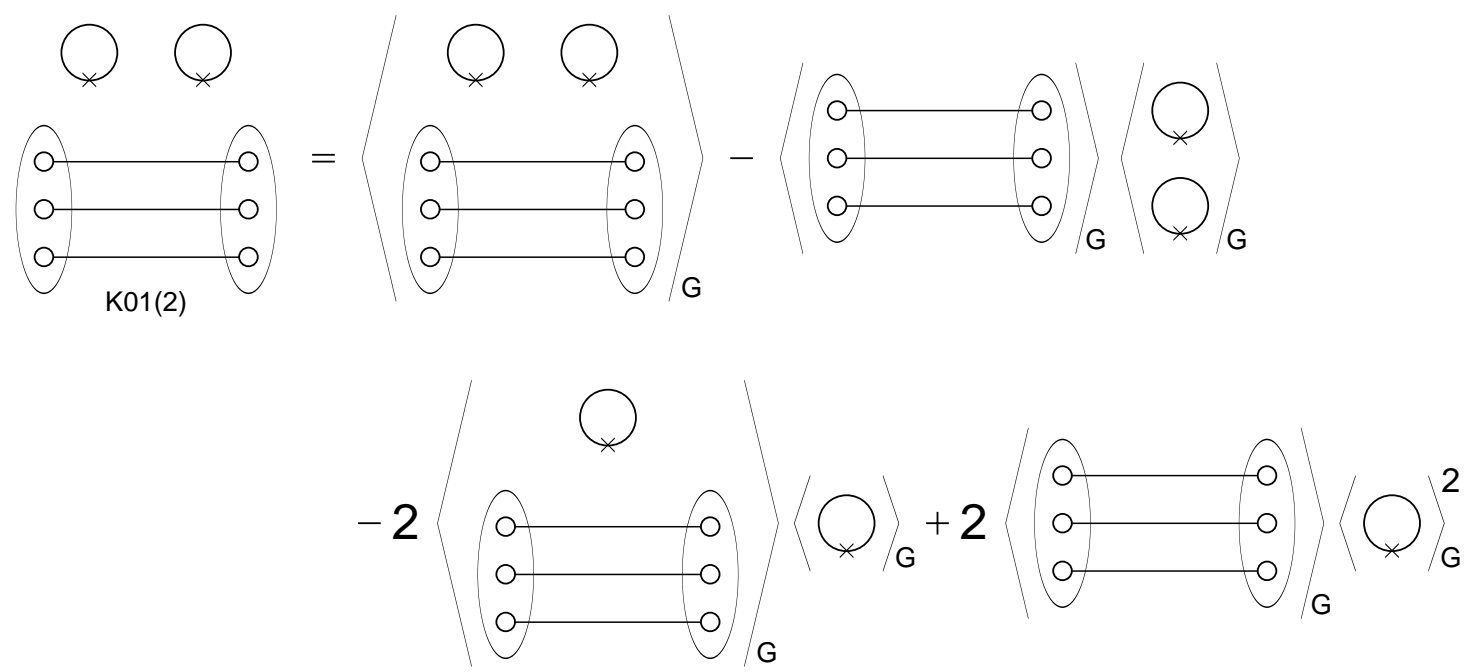

Note that the naming of the different contributions is intended to be mnemonic. The initial character differentiates between contributions of varying number of disconnected parts; $I$ denotes connected diagrams, $J$ disconnected ones with two parts and $K$ the disconnected diagram with three parts. The digit following the initial character indicates the power of the external electric field contributed specifically by the source and sink smearings. The next digit is simply a running index numbering the contributions in each class. Finally, as already mentioned under item e. above, the integer in the parentheses denotes the number of individual contributions from the Wick expansion of (32) summarized by the diagram. 


\subsection{Calculational details}

The code written to compute the diverse diagrams in Fig. 1 1 relied heavily on the Chroma Library for Lattice Field Theory $[22,23]$. In practice, the propagator chains connecting neutron source and sink were calculated in sequential fashion. Starting from a spacetime location $x$ and a specific set of color, Dirac and flavor indices $a, \alpha$ and $f$, as well as choosing the desired order $i$ of the smearing in the external field, one constructs the smeared source vector $\delta_{\beta \alpha} P_{i}^{\dagger(f)}(y, x)$ using the appropriate iterative procedure (27), (28) or (29). While no loss of generality is incurred by performing the calculation for only one particular $x$, all combinations of the other indices are ultimately required for the contractions at the neutron source (of course, different flavors are related in a trivial manner). Propagating the aforementioned specific smeared source vector yields directly the smeared-to-point propagator $K_{\gamma \alpha}^{(f) c b}(z, y) P_{i}^{\dagger(f)}(y, x)$. A vertex insertion implies multiplication with the corresponding matrix $M_{j}$, yielding a new source vector $M_{j \delta \gamma}^{(f) d c}(w, z) K_{\gamma \alpha}^{(f) c b}(z, y) P_{i}^{\dagger(f)}(y, x)$. This source vector is then again propagated, thus building up the propagator chain sequentially. When finally arriving at the neutron sink, the appropriate sink smearing is applied, using again (27), (28) or (29).

The disconnected quark loops were evaluated using stochastic estimation. To estimate the trace over all indices implied by the loop, a basis of 120 stochastic sources (240 for two cases of external fields which engender particularly strong statistical fluctuations, cf. section 4.2) was used. Again, starting at each stochastic source, propagator chains were constructed sequentially, and finally contracted again with the stochastic source. The sources were complex $Z(2)$ sources, distributed homogeneously over spacetime, Dirac and color spact5, i.e., each point in that product space was associated with a value from the set

$$
\{1+i, 1-i,-1+i,-1-i\}
$$

with equal probability.

Disconnected contributions exhibit strong statistical fluctuations, and two possibilities of reducing these fluctuations were investigated. On the one hand, the consequences of only switching on the external electric field a short time before the introduction of the neutron source and switching it off soon after the annihilation by the neutron sink

\footnotetext{
${ }^{4}$ Note that the positions of the interaction vertices in the diagrams in Fig. 1 1 are not external parameters, but integration variables. Thus, e.g., diagram I01(12) does not represent a full four-point function, but only a very specific space-time integral over a four-point function. It is these integrations which render the calculation tractable by the sequential procedure described here; they provide precisely the contraction between a vertex and an attached propagator which permits treating an inserted vertex simply as one single new source, devoid of external parameters and spread out over all of space-time, to be submitted to the subsequent propagation.

${ }^{5}$ For the flavor $S U(3)$-symmetric ensemble $m_{u}=m_{d}=m_{s}$ used in this work, it is sufficient to consider one flavor and weight the result by the appropriate combination of fractional charges to obtain the full value of the loop diagram.
} 

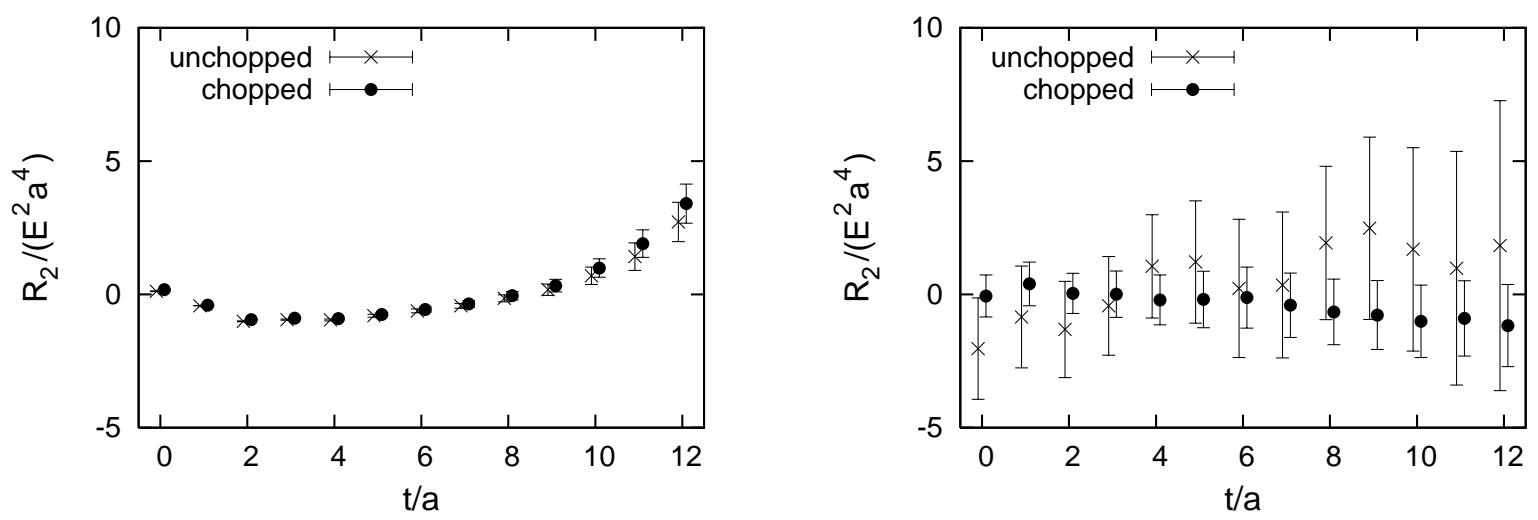

Figure 2: Comparison of results obtained using chopped and unchopped external fields, as described in the main text. Left panel displays the sum of the connected diagrams $I 01$, $I 02$ and I03; right panel the sum of the disconnected diagrams $J 01$ and J03. Results are shown as a function of temporal source-sink separation, in each case normalized by the neutron two-point function in the absence of the external field, i.e., shown are the contributions by the respective subsets of diagrams to the ratio $R_{2}$ defined in eq. (40). All measurements are taken at integer times; data are slightly displaced from those times in the figures for better readability. The electric field $E$ providing the scale is cast in Gaußian units. Shown are unrenormalized raw data, i.e., for the purpose of this comparison, $z_{V}=1$ in the vertices $(\underline{(24)}),(25)$.

were explored. This procedure will be referred to as "chopping" the external field in the following. It is motivated by the expectation that, if sufficient time has elapsed between the introduction of the neutron source and the neutron mass measurement to filter out the true neutron ground state, then also any switching-on effects generated prior to the introduction of the neutron source will have decayed. However, the statistical fluctuations of disconnected diagrams will be significantly affected by chopping the external field. Summing up contributions due to the coupling of the external field to vacuum fluctuations far in the past or the future of the neutron mass measurement, while not expected to influence the outcome of the latter, will certainly add statistical noise to it. Chopping the external field can reduce that noise significantly by discarding irrelevant vacuum fluctuations. This was tested using the external field $A_{3}=E\left(t-t_{0}\right)$ with $t_{0}=-10 a$, where here and in the following, the temporal lattice boundaries are located at $t=-10 a$ and $t=22 a$, and the neutron source is located at $t=0$. Figs. 2 and 3 compare results obtained without chopping, i.e., $A_{3}=E\left(t-t_{0}\right)$ throughout the lattice, with results obtained by setting $A_{3}=E\left(t-t_{0}\right)$ only for $-a \leq t \leq 14 a$ and $A_{3}=0$ for other times. As expected, no significant differences arise in the measured correlator ratio. This is particularly clear in the connected contributions, which are determined very accurately; in the disconnected contributions, a significant reduction 


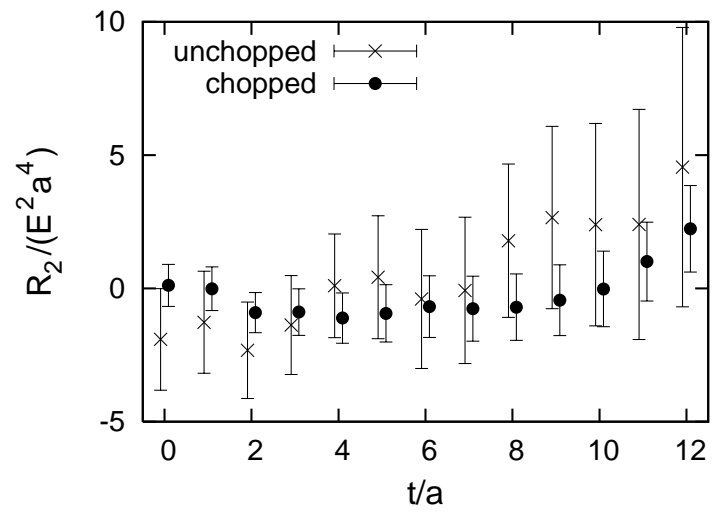

Figure 3: Comparison of results obtained using chopped and unchopped external fields, analogous to Fig. 2, but showing the contributions of the connected diagrams I01, I02, $I 03$ and the disconnected diagrams J01, J03 in one figure.

of the statistical uncertainty results. Note that the neutron mass shift is ultimately extracted specifically from the slope of the correlator ratio shown in Figs. 2 and 3 , as discussed in section 3. Due to the advantages offered by chopping the external field, all further measurements reported in the following, cf. in particular section 4.2, were obtained using chopped external electric fields.

On the other hand, a further possibility of reducing the uncertainty of stochastic estimation which was explored is dilution [24], specifically dilution in the Dirac index. In other words, besides the stochastic estimation scheme described above, also an alternative scheme was considered in which each value of the Dirac index in the loop trace was considered separately, with $Z(2)$ sources distributed homogeneously only over space-time and color space in each case, the sum over those values yielding the Dirac trace at the end. The comparison between the two schemes was carried out for the external field $A_{3}=E t$, chopped as described further above, with the neutron source again located at $t=0$. Fig. 4 shows the respective results obtained for the disconnected diagrams $J 01$ and J03. Evidently, for this particular external field and dilution scheme, there is no computational advantage in dilution; the statistical uncertainty in fact is slightly larger in the diluted case. As a consequence, dilution was not considered any further in the present investigation; this does not exclude that a comprehensive survey of various implementations of dilution could yield computationally more advantageous schemes.

\section{Interpretation of the neutron two-point function}

The standard method of extracting ground-state hadron masses is to project the hadron two-point function onto a definite momentum, consider an appropriate Dirac component, 

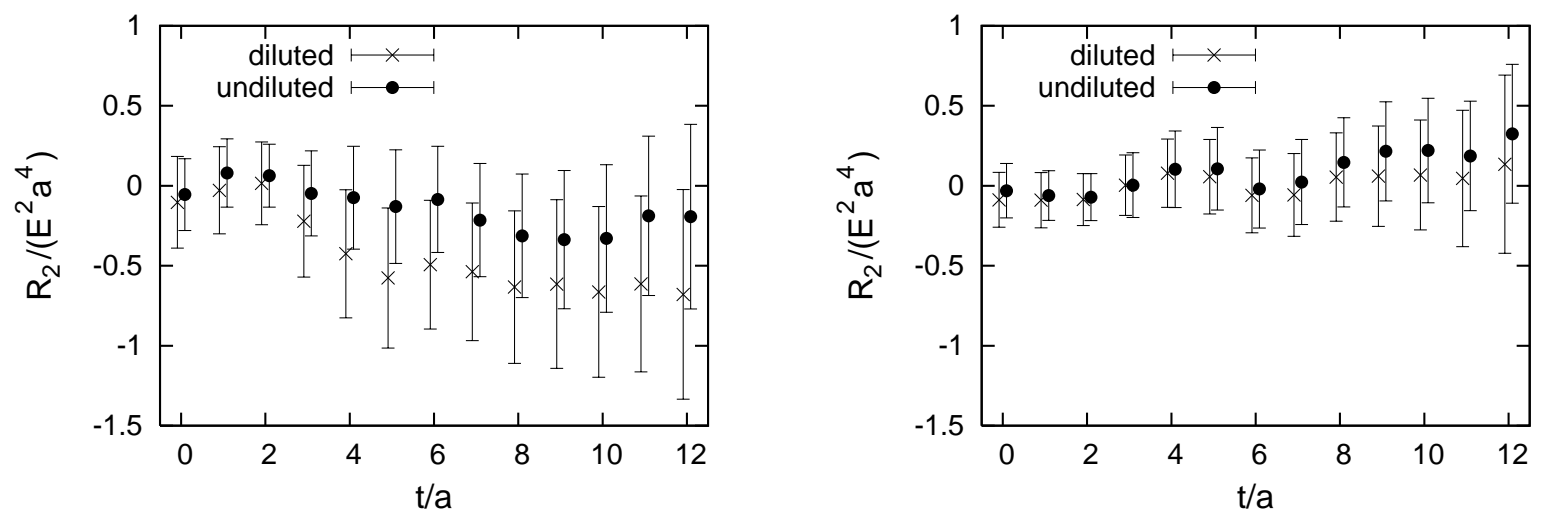

Figure 4: Comparison of results obtained using undiluted and diluted stochastic sources, as described in the main text. Diagram $J 01$ (left) and diagram $J 03$ (right) are displayed as a function of temporal source-sink separation, each normalized by the neutron twopoint function in the absence of the external field, i.e., shown are the individual contributions by the two diagrams to the ratio $R_{2}$ defined in eq. (40). All measurements are taken at integer times; data are slightly displaced from those times in the figures for better readability. The electric field $E$ providing the scale is cast in Gaußian units. Shown are unrenormalized raw data, i.e., for the purpose of this comparison, $z_{V}=1$ in the vertices $(24),(25)$.

and compare the measured data to the corresponding spectral representation. Choosing, specifically, zero momentum and unpolarized neutron states,

$$
G(p=0, t)=\int d^{3} x^{\prime} \operatorname{Tr}\left(\frac{1+\gamma_{0}}{2}\left\langle N\left(x^{\prime}\right) \bar{N}(x)\right\rangle\right) \longrightarrow W \exp (-m t)
$$

for sufficiently large times $t$, where the neutron source location defines $t=0$, and $W$ characterizes the overlap between the state created by the operator $\bar{N}$ and the true neutron ground state. Thus, the neutron mass $m$ can be extracted from the exponential decay of the correlator (35).

Furthermore, if one is calculating the correlator as a function of a small external parameter, such as an external electric field $E$, one can expand in $E$,

$$
\begin{aligned}
& m=m_{0}+m_{1} E+m_{2} E^{2}+O\left(E^{3}\right) \\
& W=W_{0}+W_{1} E+W_{2} E^{2}+O\left(E^{3}\right)
\end{aligned}
$$

and then the Taylor expansion of (35) contains the quadratic term

$$
G^{(2)}(p=0, t)=\exp \left(-m_{0} t\right)\left[W_{2}-W_{0} m_{2} t-W_{1} m_{1} t+W_{0} m_{1}^{2} t^{2} / 2\right] E^{2} .
$$


Using the fact that the neutron's electric dipole moment vanishes, $m_{1}=0$, and dividing by the correlator $G_{0}$ obtained in the absence of the external field,

$$
G_{0}(p=0, t) \longrightarrow W_{0} \exp \left(-m_{0} t\right)
$$

one has

$$
R_{2}(t) \equiv \frac{G^{(2)}(p=0, t)}{G_{0}(p=0, t)} \longrightarrow\left(\frac{W_{2}}{W_{0}}-m_{2} t\right) E^{2},
$$

allowing one to extract the neutron electric polarizability

$$
\alpha=-2 m_{2},
$$

cf. (11), from the slope of (40) as a function of $t$.

Two assumptions underlie this procedure, namely, time-independence of the Hamiltonian and spatial translational invariance. As already indicated in section 1, neither of the external gauge fields (2) and (3) investigated in the present work satisfies both of these assumptions simultaneously. As a result, the standard analysis discussed above, which would be appropriate in infinitely extended space-time, needs to be reconsidered in more detail.

\subsection{Temporally varying gauge field}

Consider first the case of the external field (2),

$$
A_{3}=E\left(t-t_{0}\right) \equiv A+E t .
$$

In this case, one does have spatial translational invariance, but there is no invariance under arbitrary temporal shifts. A translation in time corresponds to a shift in the constant component $A$ of the gauge field, and, on a space of finite extent, different $A$ are in general physically inequivalent, since only gauge transformations which shift $A$ by certain finite increments exist. Therefore, the Hamiltonian in the presence of the field (42) is time-dependent 6 (with a periodicity which depends on the strength of the electric field $E$ ).

These observations affect the above analysis in two ways. For one, the correlator (35) really depends on two external parameters, $E$ and $A$. As a consequence, the correlator ratio (40) contains all quadratic dependences which can result in the presence of the two parameters,

$$
R_{2}(t) \longrightarrow \frac{1}{W_{0}}\left(W_{2}^{A A} A^{2}+W_{2}^{A E} A E+W_{2}^{E E} E^{2}\right)-\left(m_{2}^{A A} A^{2}+m_{2}^{A E} A E+m_{2}^{E E} E^{2}\right) t .
$$

\footnotetext{
${ }^{6}$ Note that one cannot argue external fields of the type (2) to be gauge-equivalent to time-independent ones such as (3) on a finite coordinate space; the corresponding gauge transformation conflicts with the boundary conditions.
} 
On the other hand, for a small external field, the ground-state neutron mass and wave function adjust adiabatically as time evolves; this implies that the overlap coefficients $W_{2}^{* *}$ (and also $m_{2}^{* *}$ ) in (43) are time-dependent, complicating the extraction of the neutron mass shift from the slope of the correlator ratio as a function of time. Even with the expanded set of data obtained within the present work, not enough information is available to disentangle these time dependences in the most general case. However, in one specific situation, which will be argued below to be the relevant one as far as the extraction of the neutron electric polarizability is concerned, the slope of $R_{2}(t)$ indeed does yield the neutron mass shift directly; namely, when the Hamiltonian is stationary in time. In that case, time dependences in the coefficients $W_{2}^{* *}$ (and $m_{2}^{* *}$ ) are relegated to higher than linear order 7 , and one can indeed equate, up to a minus sign, the slope of $R_{2}(t)$, cf. (43), with the mass shift

$$
\Delta m=m_{2}^{A A} A^{2}+m_{2}^{A E} A E+m_{2}^{E E} E^{2} .
$$

Moreover, since a shift in $A$ is equivalent to a shift in time, stationarity of the Hamiltonian in time also implies stationarity in $A$, i.e., the mass shift (44) (and consequently the slope of $\left.R_{2}(t)\right)$ is stationary in $A$ in this particular situation. Thus, in analyzing the measured data below, the slope of $R_{2}(t)$,

$$
S_{2}=\frac{d R_{2}}{d t}
$$

will be extracted 8 and, for given $E$, the unique external field will be sought out at which $S_{2}$ is stationary with respect to $A$. Since this is then necessarily the point at which the Hamiltonian is stationary in time, at that point, then, one can identify

$$
S_{2}=-\Delta m \text {. }
$$

\footnotetext{
${ }^{7}$ As already mentioned at the end of section 2.1, and discussed further in section 4.2, here, an additional technical issue arises: While stationarity of the Hamiltonian guarantees that the neutron wave function is stationary, there is, in addition, a time dependence contained in the smeared neutron sink via eqs. (28), (29). As a result, the overlap between neutron wave function and sink can still contain contributions linear in time if one insists on manifest invariance of the neutron sink with respect to gauge transformations of the external gauge field, implying the inclusion of (28), (29) in the smeared sink construction. On the other hand, if one restricts the calculation to the fixed gauge field (42) and foregoes manifest invariance of the neutron sink with respect to gauge transformations of the external field, it is legitimate to use the time-independent smeared sink (27) alone. In terms of the diagrammatic representation of Fig. 1, this corresponds to discarding all diagrams involving smeared sinks other than $\bigcirc$. In the analysis below, both options will be treated, and the final result for the neutron electric polarizability will be seen to be uninfluenced by this choice. A way to avoid this issue, not explored within the present investigation, would be to use a point neutron sink; such a sink would be simultaneously time-independent and invariant under gauge transformations of the external field. On the other hand, a point sink would have a small overlap with the true neutron wave function, implying a lessened efficiency in the extraction of the neutron ground state signal.

${ }^{8}$ In practice, the average slope over a fixed measurement time interval will be determined in order to reduce the statistical uncertainty.
} 
To complete the analysis, it is necessary to discuss in more detail the dependence of the neutron mass shift $\Delta m$ on the parameters $E$ and $A$, and, in particular, the relevance of stationarity in $A$. In general, the part of the neutron mass shift which is of second order in the external gauge field (42) can be written as a quadratic form in the parameters $E$ and $A$, cf. (44). However, this quadratic form is not yet defined unambiguously and its coefficients can consequently not yet all be interpreted as bona fide physical properties of the neutron. To see this, consider shifting the entire neutron mass measurement process by a time increment $\bar{t}$, i.e., the neutron source, which starting with (35) has so far been assumed to be located at $t=0$, shall, for the sake of the following argument, now be located at $t=\bar{t}$. If one concomitantly introduces a shifted time coordinate $t^{\prime}=t-\bar{t}$ and a shifted

$$
\bar{A}=A+E \bar{t},
$$

then, in terms of the shifted quantities, the problem takes a form identical to the original one, i.e., one measures the mass shift

$$
\begin{aligned}
\Delta m & =m_{2}^{A A} \bar{A}^{2}+m_{2}^{A E} \bar{A} E+m_{2}^{E E} E^{2} \\
& =m_{2}^{A A} A^{2}+\left(m_{2}^{A E}+2 \bar{t} m_{2}^{A A}\right) A E+\left(m_{2}^{E E}+\bar{t} m_{2}^{A E}+\bar{t}^{2} m_{2}^{A A}\right) E^{2} \\
& =\bar{m}_{2}^{A A} A^{2}+\bar{m}_{2}^{A E} A E+\bar{m}_{2}^{E E} E^{2} .
\end{aligned}
$$

Thus, in terms of the original definition of $E$ and $A$, cf. (42), the shifted measurement yields a quadratic form for the mass shift with different coefficients $\bar{m}_{2}^{A E}$ and $\bar{m}_{2}^{E E}$ (whereas the remaining coefficient is invariant, $\bar{m}_{2}^{A A}=m_{2}^{A A}$ ). Therefore, the question arises how the neutron electric polarizability is to be extracted from the total mass shift $\Delta m$; evidently, polarizability effects enter both the coefficients $\bar{m}_{2}^{E E}$ and $\bar{m}_{2}^{A E}$, which can be traded off against one another, as demonstrated above.

As a first step towards disentangling the different effects at play, the immutable character of the coefficient $\bar{m}_{2}^{A A}$ should be noted, which allows it to be interpreted as an unambiguous property of the neutron. This property moreover is separate from the electric polarizability; as verified by explicit calculation below, also at $E=0$ one obtains the mass shift $\Delta m=\bar{m}_{2}^{A A} A^{2}$, encoding the response of the neutron to distortion by the presence of the constant background field9. With $\Delta m=\bar{m}_{2}^{A A} A^{2}$ representing the response of a neutron already in the absence of any external electric field, one would indeed expect modifications of this response due to a distortion of the neutron by an additional electric field to occur only at higher than quadratic order in the external gauge field. The representation-independence of $\bar{m}_{2}^{A A}$, i.e., its independence of the choice of $\bar{t}$, thus seems plausible, and is consistent with the interpretation of the $\bar{m}_{2}^{A A} A^{2}$ term as a response separate from the electric polarizability.

\footnotetext{
${ }^{9}$ Note that the effect of such a constant background field is equivalent to a modification of the boundary conditions in the relevant direction, introducing nontrivial Bloch momenta varying with quark flavor.
} 
On the other hand, in view of (49), there is one special representation, i.e., choice of $\bar{t}$, which seems particularly transparent, namely, the representation in which $\bar{m}_{2}^{A E}$ vanishes, such that 10

$$
\Delta m=\bar{m}_{2}^{A A} A^{2}+\bar{m}_{2}^{E E} E^{2} .
$$

The form (51) suggests an interpretation of the data in terms of two, now cleanly disentangled effects, namely, the polarizability effect determined by $\bar{m}_{2}^{E E}$ and the effect of introducing a constant background field, embodied in the coefficient $\bar{m}_{2}^{A A}$. Thus, in this special representation, the electric polarizability is given by

$$
\alpha=-2 \bar{m}_{2}^{E E} .
$$

In other words, to isolate the electric polarizability effect from the complete mass shift, one simply sets $A=0$ in the representation (51).

Finally, it is possible to rephrase this prescription for extracting the neutron electric polarizability in a manner which is independent of the particular representation, i.e., the choice of $\bar{t}$. Setting $A=0$ in the representation (51) is tantamount to evaluating the mass shift $\Delta m$ at the extremum in $A$. However, this way of stating the prescription does not rely on that specific representation; after all, in view of (48) and (50) in conjunction with (47), different representations are related by shifting the value of $A$, and the extremum of $\Delta m$ as a function of $A$ is invariant under such shifts. Thus, one can isolate the neutron electric polarizability in any and all representations by seeking out the stationary point of the mass shift as a function of $A$.

In view of this, and the equivalence of shifts in $A$ with shifts in time, the neutron electric polarizability can indeed be extracted by considering the correlator ratio $R_{2}(t)$, cf. (43), specifically for external gauge fields in the vicinity of which the Hamiltonian is stationary in time; this validates the arguments presented further above in conjunction with eqs. (44)-(46).

\subsection{Spatially varying gauge field}

In the case of the gauge field (3),

$$
A_{0}=-E x_{3},
$$

\footnotetext{
${ }^{10} \mathrm{~A}$ way to understand how the simplified dependence (51) arises is the following: Choosing $\bar{t}$ such as to realize (51) shifts the mass shift measurement time interval towards the time $t=0$. Now, in view of the definition (42), the time $t=0$ is special in that the $A$ - and $E$-directions in parameter space are, in a sense, orthogonal there: At $t=0$, a change of $E$ affects only the slope of $A_{3}$, but not its value; at other times, this is not the case and a change in $E$ also implies an adjustment of the value of $A_{3}$ itself, which could be equally effected (or compensated) by a change in $A$. It is this implicit relation between $E$ and $A$ which generates the coupled dependence (44); however, if one measures near $t=0$, the implicit relation is dissolved and it is natural to obtain the decoupled dependence given by (51).
} 
one does have a time-independent Hamiltonian, and consequently one can straightforwardly extract the energy of the neutron ground state from the exponential time decay of the neutron two-point function. However, this invariance under translations in time comes at the expense of breaking spatial translational invariance. The linear dependence of (53) on $x_{3}$ conflicts with the periodic boundary conditions; when traveling through the lattice in the 3-direction, as the boundary is traversed, $A_{0}$ is forced to jump, implying a spike in the electric field which is present in addition to the constant electric field induced by (53) .

Therefore, the neutron is not propagating in a spatially homogeneous background and its momentum is not a good quantum number11. As a result, the ground state energy one extracts from the decay of the neutron two-point function contains not only the desired mass shift associated with the electric polarizability, but further contributions due to, e.g., the effective movement in a spatially varying potential and additional distortions of the neutron by the electric field spikes. Within the present investigation, no prescription for disentangling the neutron electric polarizability from these other effects with a level of cogency comparable to the one discussed in the previous section emerged. Nevertheless, the ground state energy obtained below using the external gauge field (53) is consistent with the polarizability mass shift obtained using the external field (42), suggesting that the contamination by the additional effects mentioned above is not dominant. At least as far as the neutron's effective propagation in a spatially varying potential is concerned, this seems plausible, since the quantum mechanical zero-point energy associated with such motion is suppressed by the comparatively large mass of the neutron.

Note that, although superficially the external fields (42) and (53) seem quite similar, and simply related by an exchange of the temporal with a spatial direction, the physical issues arising in the two cases are quite distinct. This is due to the way the mass measurement is set up. Up to exponentially suppressed effects, the neutron mass is determined by physics within a limited time interval, between neutron source and sink. The temporal boundaries, located far in the past or the future of the measurement, have a negligible effect on the latter. By contrast, one cannot similarly contain the region relevant for the measurement in the spatial directions. In situations with spatial translational invariance, by projecting onto a definite momentum, one explicitly weights all of space equally during the entire measurement process. Even in the absence of spatial translational invariance, it is up to the dynamics to determine whether there is a significant probability of finding the neutron near the spatial boundary. Thus, in general, the spatial boundary conditions have a crucial influence on the problem.

In the case of the external field (42) discussed in the previous section, this entails that shifts of the gauge field $A_{3}$ by a constant $A$ have a physical effect, since gauge

\footnotetext{
${ }^{11}$ It is, of course, still legitimate to use a zero-momentum neutron sink, as in (35), since it will presumably have a finite overlap with the true neutron ground state wave function. However, that wave function itself will not carry a definite momentum.
} 
transformations designed to remove such a shift conflict with the spatial boundary conditions. As a consequence, physics varies locally with time, as discussed extensively further above. On the other hand, the neutron mass measurement is insensitive to the behavior of the external field at the temporal boundaries.

In the case of the external field (53), one encounters a largely converse situation: The neutron ground state is sensitive to the spatial boundary, at which it encounters spikes in the external electric field; on the other hand, as long as one is not in the vicinity of the boundary, physics does not vary locally in space. The latter is due to the fact that one can indeed remove constant shifts in the field $A_{0}$ in the time interval relevant for the neutron mass measurement using gauge transformations. These transformations do of course need to exhibit additional nontrivial structures located far in the past and the future of the measurement, but these structures will not influence the measurement. This also motivates the fact that no explicit freedom of shifting $A_{0}$ by a constant is included in (53), in contradistinction to (42). Such shifts are not expected to yield new physics according to the above argument.

Comparing the two cases, ultimately (42) can be treated in a more satisfactory fashion because the positioning of the neutron source and sink allows one to contain and control the breaking of temporal translational invariance introduced by the field (42). By contrast, in the case of (53), there is no analogous control; the neutron dynamics must be allowed to explore space and, in general, the breaking of spatial translational invariance will influence the measurement in a nontrivial fashion.

\section{Measurement results}

\subsection{Quark wave function renormalization}

To determine the renormalization factor $z_{V}$ in (24), (25), a measurement of the number of valence quarks in the neutron was carried out and subjected to the condition that this number equal three. In practice, this is realized by measuring the appropriate three-point function, i.e., a diagram of the type $I 03$, with the difference that the lone operator insertion is of the form of $M_{1}$, cf. (24), without the weighting by the quark electric charge $q_{f}$, and with a formal external gauge field

$$
A_{0}(x)=\delta\left(x_{0}-t\right)
$$

where $t$ is a time between neutron source and sink. Normalizing this by the neutron two-point function yields, up to an additional factor $i$ stemming from the Euclidean treatment of the time coordinate, the (lattice analogue of the) expectation value of $\int d^{4} x j_{0} A_{0}$ in the neutron, where $j_{0}$ denotes the temporal component of the quark current. In view of (54), this reduces to the number of (valence) quarks $n=\int d^{3} x j_{0}$ present at the time $t$. Fig. 5 displays the plateau obtained measuring $n$ at different insertion times 


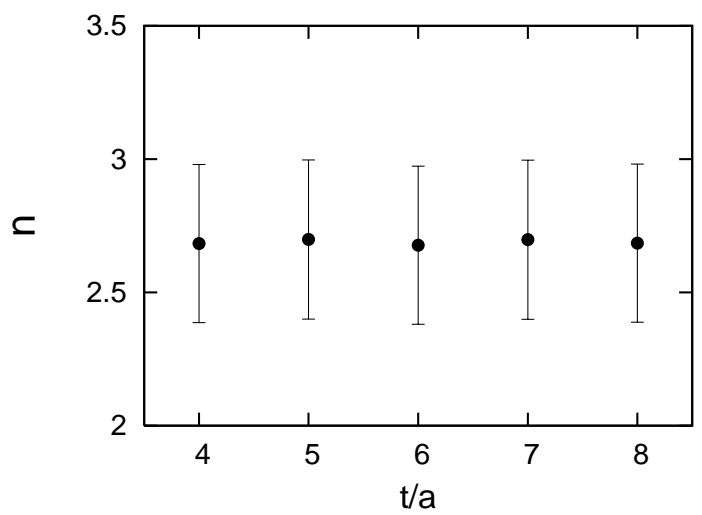

Figure 5: (Unrenormalized) number of valence quarks $n$ in the neutron measured for a range of insertion times. The neutron source is located at $t=0$ and the neutron sink at $t=13 a$.

$t$ for fixed neutron source and sink. Taking the average of the displayed plateau values, one infers

$$
z_{V}=1.12 \pm 0.12
$$

where the uncertainty was obtained using the jackknife method. This measurement of $z_{V}$ enters all further measurements below; its uncertainty will be jackknifed into those measurements.

\subsection{Neutron mass shift}

Measurements of the neutron mass shift according to the discussion in section 3 were taken for the following cases: With the location of the neutron source once again defining $t=0$, the external gauge field (42) was studied for the cases $t_{0}=-10 a, t_{0}=0$ and $t_{0}=6 a$ (where $a$ denotes the lattice spacing) at a fixed nonvanishing value of $E$. Furthermore, the case $E=0$ at a fixed nonvanishing value of $A$ in (42) was investigated. The external gauge field (53) was treated for a fixed nonvanishing value of $E$, where the plane $x_{3}=0$ was taken to define locations maximally distant from the lattice boundary in the 3-direction, and was simultaneously used as the location of the neutron source (i.e., the smeared quark sources were constructed using an initial position $x$ in (21) located in the $x_{3}=0$ plane).

Furthermore, as discussed in section 2.3, to suppress fluctuations in disconnected diagrams, these external gauge fields were chopped in the time direction, i.e., $A_{3} \equiv 0$ and $A_{0} \equiv 0$ for $t<-a$ and $t>14 a$ in the following. Only for $-a \leq t \leq 14 a$ do $A_{3}$ and $A_{0}$ take the forms (42) and (53), respectively. The temporal boundaries of the lattice, at which Dirichlet boundary conditions are enforced on the quark fields, are located at $t=-10 a$ and $t=22 a$. Stochastic estimation of the disconnected diagrams was based 
on 120 stochastic sources, as described in section 2.3, except for the cases $t_{0}=-10 a$ and $E=0$ in (42), for which 240 stochastic sources were used.

Figs. 6 10 display measurements of the ratio

$$
R_{2}(t) \equiv \frac{G^{(2)}(p=0, t)}{G_{0}(p=0, t)}
$$

cf. (40), (43), for all the aforementioned external gauge fields, in units of the relevant external field magnitude. That is, $R_{2}$ is shown in units of $A^{2}$ for the case $E=0$ and in units of $E^{2}$ in the other cases; furthermore, here and in the following, Gaußian units are adopted. Different subsets of diagrams from Fig. 1 contributing to $R_{2}$ are shown in the individual plots (a)-(d) in each case. Figs. 6-10 (a) show only the contributions from connected diagrams with lowest-order smearing, i.e., the diagrams $I 0 *$. Figs. 6 10 (b) show the result of including all connected diagrams, $I * *$. Note that, in the case of the external field (53), there are no connected contributions beyond $I 0 *$, since smearing occurs only in the spatial directions and thus never involves the gauge field component $A_{0}$. Figs. 6-10 (c) show the result of including all diagrams with lowest-order smearing, i.e., the diagrams $* 0 *$. Finally, Figs. 6-10 (d) show the sum of all diagrams depicted in Fig. 1. Note that, in the $S U(3)$ flavor-symmetric case investigated in this work, the only nonvanishing disconnected diagrams are $J 01$ and $J 03$, regardless of the external field used. This is due to the fact that the disconnected loop with a linear external field insertion is proportional to the sum of the quark charges, and therefore vanishes. Thus, Figs. 6-10 (c) and Figs. 610 (d) contain the same disconnected contributions.

In comparing Figs. 6 8, which display the results obtained using the external gauge field (42) for various $t_{0}$, the different vertical scales should be noted. Compared to the case $t_{0}=-10 a$, the cases $t_{0}=0$ and $t_{0}=6 a$ exhibit only very small slopes, which are determined with relatively small uncertainties, cf. also Table 1 below. The foremost observation to be drawn from Figs. 6 , 8 is that the slope of $R_{2}$ indeed depends sensitively on $t_{0}$, or, equivalently, the constant offset $A$ in the external gauge field (42), as expected. This is also corroborated by the $E=0$ measurement displayed in Fig. 10. As explained in section 3.1, these measurements taken together will make it possible to disentangle the constant field effect from the electric polarizability. Before proceeding towards this central goal, a few further remarks about the data are in order.

For one, there is a strong cancellation between the diagram I02 and the corresponding contact term I03, cf. Fig. 11, The contact term I03, which originates from expanding the gauge link variables to second order in the external field, cf. (17),(22),(25), is not negligible, as a naive continuum limit might suggest; rather, it contributes to the renormalization of the $I 02$ diagram. Presumably, in the continuum limit, it would be admissible to disregard diagrams such as I03, at the expense of having to consider a strong renormalization of the diagram $I 02$ as its two vertices are permitted to approach each other. 

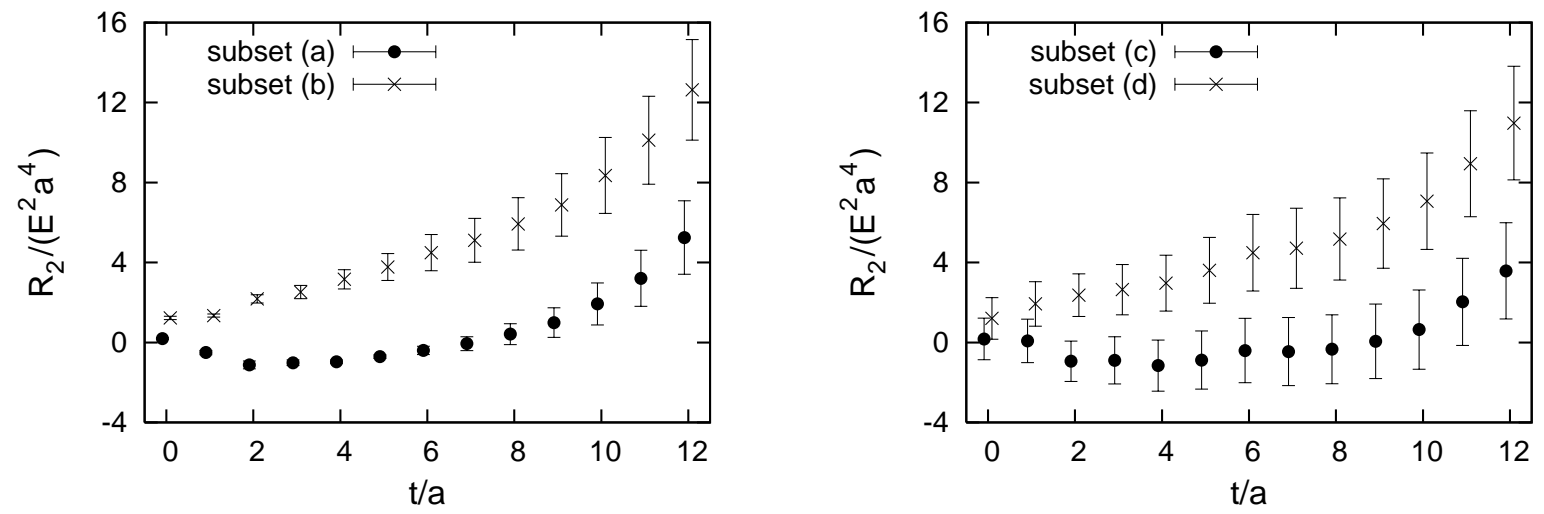

Figure 6: Contributions by selected subsets of diagrams, as specified in the main text, to the ratio $R_{2}$, as a function of temporal source-sink separation $t$. All measurements are taken at integer times; data are slightly displaced from those times in the figures for better readability. These results were obtained using an external field of the form (42), i.e., $A_{3}=E\left(t-t_{0}\right)$, with $t_{0}=-10 a$, where $t=0$ corresponds to the neutron source location. The electric field $E$ providing the scale is cast in Gaußian units.
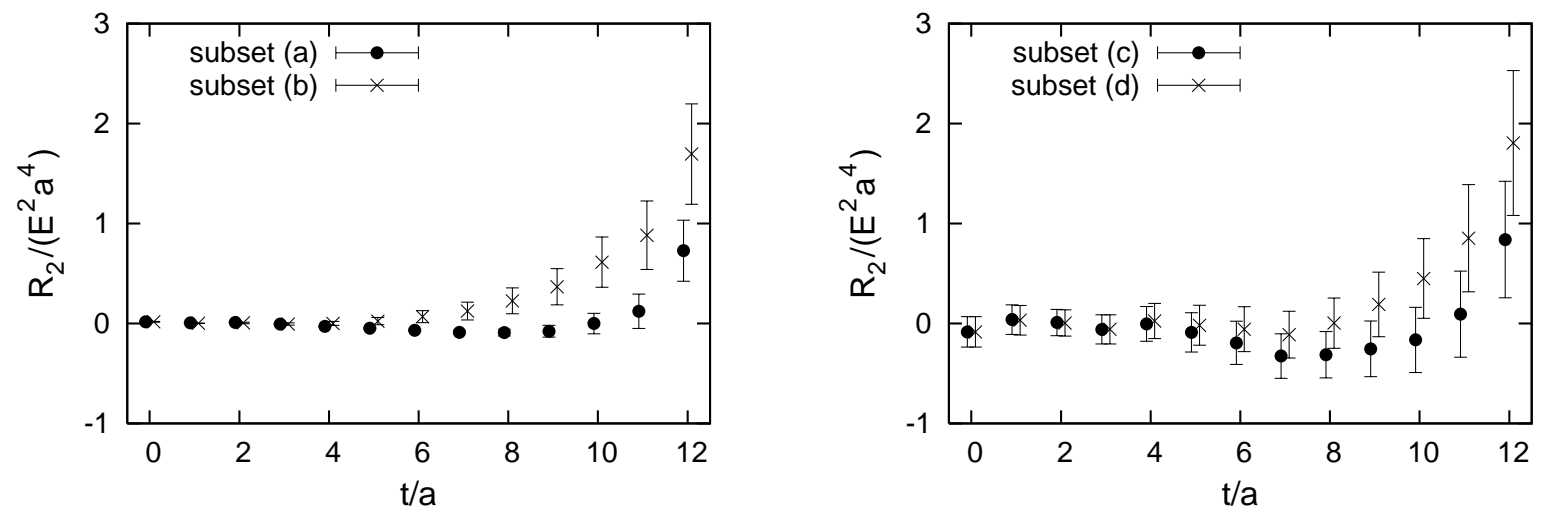

Figure 7: Contributions by selected subsets of diagrams, as specified in the main text, to the ratio $R_{2}$, as a function of temporal source-sink separation $t$. All measurements are taken at integer times; data are slightly displaced from those times in the figures for better readability. These results were obtained using an external field of the form (42), i.e., $A_{3}=E\left(t-t_{0}\right)$, with $t_{0}=0$, where $t=0$ corresponds to the neutron source location. The electric field $E$ providing the scale is cast in Gaußian units. 

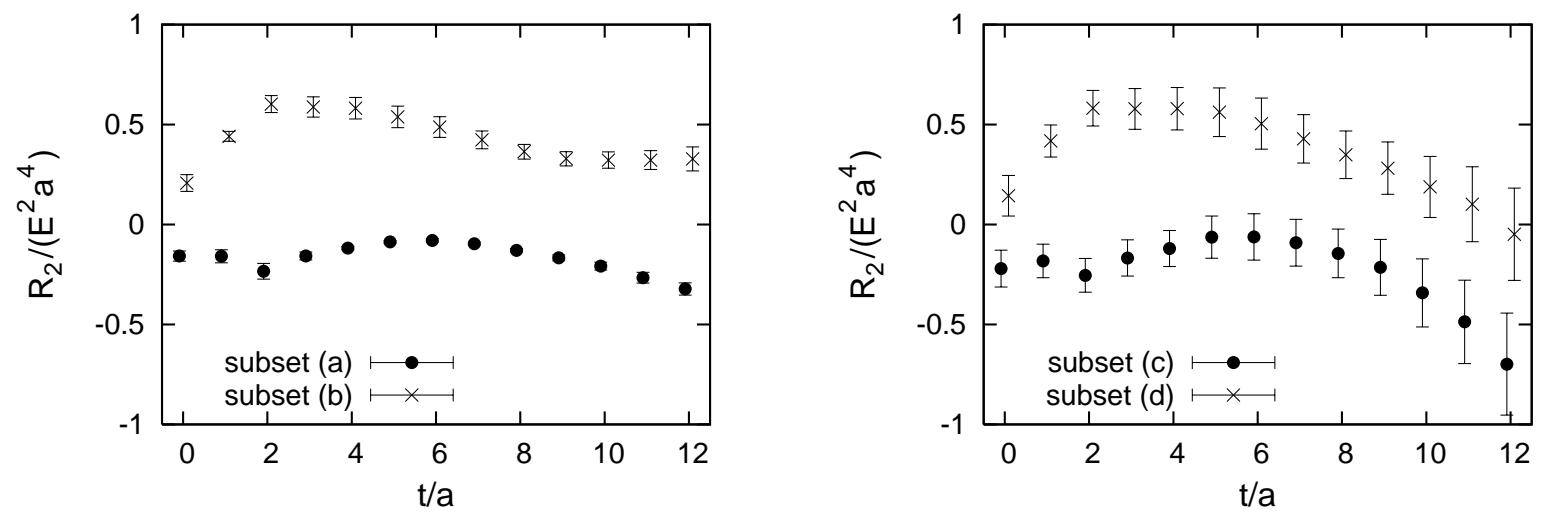

Figure 8: Contributions by selected subsets of diagrams, as specified in the main text, to the ratio $R_{2}$, as a function of temporal source-sink separation $t$. All measurements are taken at integer times; data are slightly displaced from those times in the figures for better readability. These results were obtained using an external field of the form (42), i.e., $A_{3}=E\left(t-t_{0}\right)$, with $t_{0}=6 a$, where $t=0$ corresponds to the neutron source location. The electric field $E$ providing the scale is cast in Gaußian units.
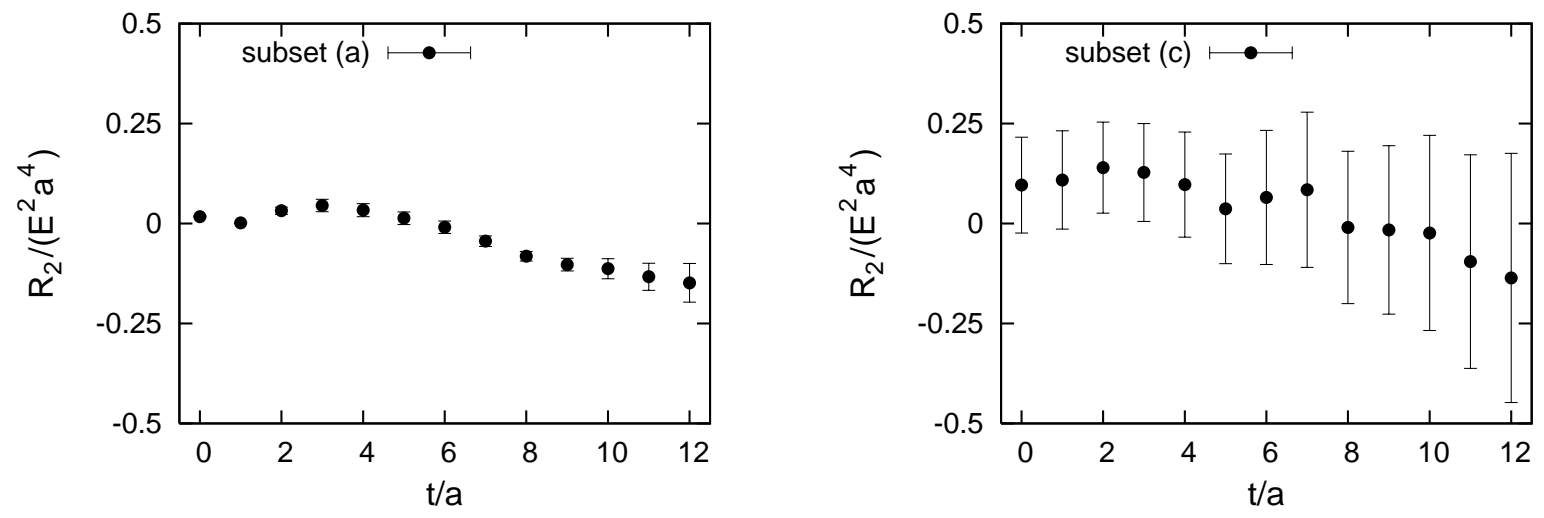

Figure 9: Contributions by selected subsets of diagrams, as specified in the main text, to the ratio $R_{2}$, as a function of temporal source-sink separation $t$. These results were obtained using an external field of the form (53), i.e., $A_{0}=-E x_{3}$; for this background, there are no smearing contributions beyond zeroth order in the external field, i.e., case (b) is identical to case (a), and case (d) is identical to case (c). The electric field $E$ providing the scale is cast in Gaußian units. 

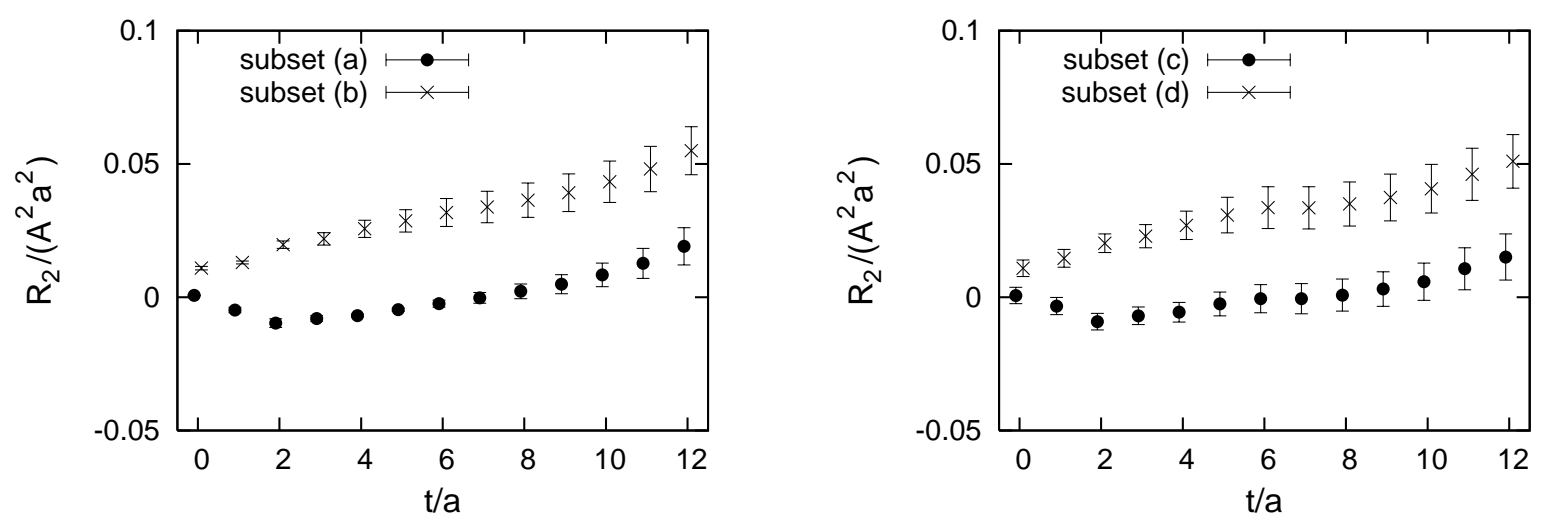

Figure 10: Contributions by selected subsets of diagrams, as specified in the main text, to the ratio $R_{2}$, as a function of temporal source-sink separation $t$. All measurements are taken at integer times; data are slightly displaced from those times in the figures for better readability. These results were obtained using an external field of the form (42) with $E=0$, i.e., $A_{3} \equiv A$. The constant background gauge field $A$ providing the scale is cast in Gaußian units.

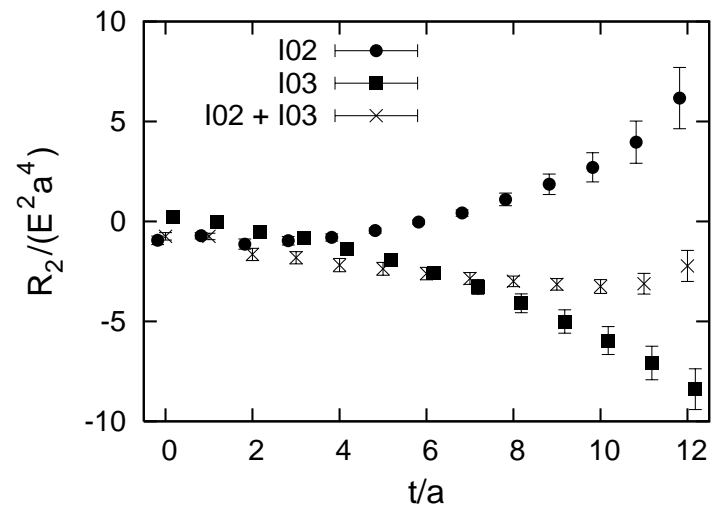

Figure 11: Contributions by diagrams I02 and I03, as well as their sum, to the ratio $R_{2}$, as a function of temporal source-sink separation $t$. All measurements are taken at integer times; data are slightly displaced from those times in the figure for better readability. These results were obtained using an external field of the form (42), i.e., $A_{3}=E\left(t-t_{0}\right)$, with $t_{0}=-10 a$, where $t=0$ corresponds to the neutron source location. The electric field $E$ providing the scale is cast in Gaußian units. 
Secondly, one can furthermore observe from Figs. 6 10 that the disconnected diagrams consistently tend to give a negative contribution to the slope of the ratio $R_{2}(t)$ for an external field of the form (42), cf. also Table 1 below. For the external field (53), the contribution is very slightly positive. However, it should be emphasized that the contributions are in no case large enough to be significant compared to the statistical uncertainty.

Thirdly, as noted in section 3.1, the higher-order (in the external field) sink smearing diagrams contained in Figs. 66 (b),(d) in general contribute additional linear time dependences to the ratio $R_{2}(t)$ beyond the ones associated with the mass shift of the neutron in the external field. Indeed, a substantial difference in slope can be seen 12 comparing Fig. 6 (a) and (b), or also Fig. 6 (c) and (d). To isolate the slope due to the mass shift itself, one should evaluate Figs. 6] (c); this comes at the expense of foregoing a form of the neutron sink (and source) which is manifestly invariant under gauge transformations of the external field. Of course, it is not imperative to use a manifestly invariant form; all that is implied by not doing so is that the neutron mass shift, a gauge-invariant quantity, has been evaluated in a specific gauge for the external electromagnetic field. Note, moreover, that this point is largely moot at any rate, since the final result for the neutron electric polarizability will be seen to not be affected significantly by the differences in intermediate data introduced by the aforementioned sink smearing effects.

Returning to the main objective, extracting the neutron mass shift from the slope of the ratio $R_{2}(t)$, Table 1 lists the slopes extracted from the data displayed in Figs. 6- 10. These slopes were obtained by performing least-square fits of linear functions in $t$ to the $R_{2}(t)$ data for a range of $t$; the uncertainties were obtained by jackknife analysis. The time range used was $4 a \leq t \leq 10 a$; this choice minimizes statistical uncertainty (by using as large a time range as possible) while still allowing for a good least-square fit by a linear form as well as a jackknife bias estimate small compared to the statistical uncertainty 13 .

According to the discussion in section 3.1, to extract the electric polarizability from the data in the first three lines of Table 1, these data should be viewed as defining a parabola in $t_{0}$, and the extremum in $t_{0}$ should be sought out (note that this is equivalent to viewing the data as defining a parabola in $A$, since $A=-E t_{0}$ and $E$ is constant). At the extremum, one can then identify the slope $S_{2}$ with the (negative) neutron mass

\footnotetext{
${ }^{12}$ By considering the diagrams individually, one can indeed verify that, as expected, the difference arises specifically due to sink smearing contributions, and not source smearing contributions.

${ }^{13}$ In the case of the external field (42) with $t_{0}=0$ and $t_{0}=6 a$, a more restricted time range improves the linear fit, as is apparent, e.g., from Fig. 8, however, as already noted above, these two instances are determined with very little uncertainty (regardless of the time range used) compared to $t_{0}=-10 a$. As a result, it is the latter case which dictates the choice of time range, which then was adopted for all cases for consistency.
} 


\begin{tabular}{|c||c|c|c|c|}
\hline & $(\mathrm{a})$ & $(\mathrm{b})$ & $(\mathrm{c})$ & $(\mathrm{d})$ \\
\hline \hline $\begin{array}{c}S_{2} /\left(a^{3} E^{2}\right) \\
(42), t_{0}=-10 a\end{array}$ & $0.46(18)$ & $0.83(24)$ & $0.26(26)$ & $0.63(30)$ \\
\hline $\begin{array}{c}S_{2} /\left(a^{3} E^{2}\right) \\
(42), t_{0}=0\end{array}$ & $0.000(16)$ & $0.096(38)$ & $-0.033(43)$ & $0.063(54)$ \\
\hline $\begin{array}{c}S_{2} /\left(a^{3} E^{2}\right) \\
(42), t_{0}=6 a\end{array}$ & $-0.017(3)$ & $-0.047(7)$ & $-0.037(25)$ & $-0.067(27)$ \\
\hline $\begin{array}{c}-\Delta m /\left(a^{3} E^{2}\right) \\
(533)\end{array}$ & $-0.027(4)$ & $-0.027(4)$ & $-0.019(41)$ & $-0.019(41)$ \\
\hline \hline $\begin{array}{c}-\Delta m /\left(a A^{2}\right) \\
(42), E=0\end{array}$ & $0.0025(8)$ & $0.0028(8)$ & $0.0017(9)$ & $0.0020(10)$ \\
\hline
\end{tabular}

Table 1: Slope $S_{2}$, cf. (45)), for different external electromagnetic fields, in the appropriate external field units and units of the lattice spacing $a$. Cases (a)-(d) correspond to the different subsets of diagrams included in the corresponding Figs. 66-10 (a)-(d), cf. main text. In the case of the background field (53), as well as for (42) with $E=0$, the slope $S_{2}$ can be directly identified with the negative mass shift, $-\Delta m$; hence the labeling of the last two lines. By contrast, the data in the first three lines must be processed further to locate the stationary point as a function of $t_{0}$, at which then $S_{2}=-\Delta m$ can be identified, cf. main text and Table 2 .

shift, $-\Delta m$. Fitting the form (cf. (44)-(46))

$$
\begin{aligned}
S_{2} / E^{2} & =-\left(m_{2}^{A A} A^{2}+m_{2}^{A E} A E+m_{2}^{E E} E^{2}\right) / E^{2} \\
& =-\left(m_{2}^{A A} t_{0}^{2}-m_{2}^{A E} t_{0}+m_{2}^{E E}\right)
\end{aligned}
$$

to the data in Table1 1 yields parabolas with the extrema and curvatures listed in Table2. The uncertainties quoted in Table 2 were again obtained using the jackknife method. The quadratic coefficient $m_{2}^{A A}$ extracted in this way agrees well with the $E=0$ values 14 listed in the last line of Table 1, providing an independent measurement corroborating the interpretation of the data advanced in section 3.1.

The central result of this work, however, is the value of the electric polarizability of the neutron, the full value of which is obtained by multiplying the mass shift quoted in column (c) of Table 2 by a factor of 2 , cf. (44),(52). In physical units, obtained by inserting $a=0.124 \mathrm{fm}$, one has

$$
\alpha=-2 \Delta m / E^{2}=(-2.0 \pm 0.9) \cdot 10^{-4} \mathrm{fm}^{3} .
$$

\footnotetext{
${ }^{14}$ The last lines of Tables 1 and 2 can be directly compared, since for $E=0$, one has $\Delta m / A^{2}=m_{2}^{A A}$, cf. (44).
} 


\begin{tabular}{|c||c|c|c|c|}
\hline & $(\mathrm{a})$ & $(\mathrm{b})$ & $(\mathrm{c})$ & $(\mathrm{d})$ \\
\hline \hline $\begin{array}{c}-\Delta m /\left(a^{3} E^{2}\right) \\
(\text { extremum) }\end{array}$ & $-0.034(6)$ & $-0.049(10)$ & $-0.052(24)$ & $-0.076(63)$ \\
\hline$-m_{2}^{A A} / a$ & $0.0027(9)$ & $0.0031(9)$ & $0.0018(16)$ & $0.0022(16)$ \\
\hline
\end{tabular}

Table 2: (Negative) mass shifts at the extrema of the parabolas defined by the data in Table 1 through the form (57), as well as the coefficient $m_{2}^{A A}$ characterizing the curvatures of the parabolas.

Note that the additional smearing contributions entering the result in column (d) indeed do not significantly alter this result. The result (58) is corroborated by the measurement using the external field (53), quoted in the fourth line of Table 1; translated into physical units, that measurement would imply a polarizability of

$$
\alpha=(-0.7 \pm 1.6) \cdot 10^{-4} \mathrm{fm}^{3} .
$$

As discussed in section 3.2, the result (59) contains systematic uncertainties (not included in the quoted statistical error) stemming from the fact that the mass shift measured in this case is contaminated by the quantum mechanical zero-point motion of the neutron and distortions of its internal wave function due to superfluous spikes in the external electric field. Thus, the result (58) is expected to be more trustworthy than the result (59). Nevertheless, the difference between the two measurements does not turn out to be significant; the aforementioned contaminations do not appear to represent appreciable effects.

Compared to the experimental value reported by the Particle Data Group [25]

$$
\alpha=(11.6 \pm 1.5) \cdot 10^{-4} \mathrm{fm}^{3}
$$

the result (58) suggests a strong variation of the electric polarizability of the neutron with the pion mass. Indeed, Chiral Effective Theory calls for such a variation [10,26-29], dominated by a $1 / m_{\pi}$ dependence at low pion masses. In the "Small Scale Expansion" approach $[27,28]$, which systematically extends leading-one-loop Heavy Baryon Chiral Perturbation Theory by including explicit $\Delta$ degrees of freedom, the electric polarizability of the neutron decreases by an order of magnitude as one varies the pion mass from the physical point up to around $400 \mathrm{MeV}$. Qualitatively, a change of sign of the polarizability at even higher pion masses, as implied by (58), does not seem implausible15, although it should be stressed that a pion mass of $759 \mathrm{MeV}$, corresponding to the dynamical quark ensemble used in the present work, is certainly far beyond the

\footnotetext{
${ }^{15}$ Of course, for very large masses, i.e., in the nonrelativistic limit, the polarizability cannot be negative due to the general properties of second-order perturbation theory.
} 
regime in which Chiral Effective Theory can be applied reliably. Lattice calculations at lower pion masses are needed in order to achieve a quantitative connection with Chiral Effective Theory.

On the other hand, the result obtained in the present work at first sight appears to be at odds with previous lattice measurements $[5,7]$. Those studies yield a neutron electric polarizability which is consistent with (60) over a wide range of (valence) quark masse: 16 , including the quark mass used in the present work. The question arises how such a weak variation with the quark mass can be reconciled with the result obtained in the present work (and also with the expectation coming from Chiral Effective Theory).

Apart from the use of the quenched approximation, the main differences between $[5,7]$ and the present treatment are that, on the one hand, $[5,7]$ work with an external field corresponding to a particular value of $t_{0}$ in (42); on the other hand, this external field is introduced into the lattice link variables in linearized form, i.e., the vertex insertion $M_{2}$, cf. (25), is not included. As discussed further above, the insertion $M_{2}$ provides contact terms which renormalize propagators with two $M_{1}$ insertions, leading to substantial cancellations; however, most importantly, the measured mass shift depends sensitively on the parameter $t_{0}$ characterizing the external field. It is instructive to reevaluate the data gathered in the present work such that diagrams generated by $M_{2}$ insertions are excluded, and at a value of $t_{0}$ corresponding to the one used in [5,7]. Two measurements are provided by [5,7]. One uses Wilson fermions with a distance of $1.7 \mathrm{fm}$ between $t_{0}$ and the mass shift measurement, yielding a polarizability 17 of $\alpha=(9.8 \pm 1.2) \cdot 10^{-4} \mathrm{fm}^{3}$ for pion masses comparable to the one at which the present work was performed; the other uses clover fermions with a distance of $1.53 \mathrm{fm}$ between $t_{0}$ and the mass shift measurement, yielding a polarizability of $\alpha=(13.9 \pm 0.8) \cdot 10^{-4} \mathrm{fm}^{3}$ at comparable pion masses. Taking into account that the mass shift measurement in the present work is centered around $t=7 a=0.87 \mathrm{fm}$, the values of $t_{0}$ corresponding to the two aforementioned cases are $t_{0}=-6.7 a$ and $t_{0}=-5.3 a$, respectively. Constructing the parabola (57) defined by excluding diagrams generated by $M_{2}$ insertions from the set of diagrams comprising case (c) above, and evaluating it at those values of $t_{0}$ yields

$$
\begin{aligned}
& \alpha\left(t_{0}=-6.7 a\right)=(20 \pm 11) \cdot 10^{-4} \mathrm{fm}^{3} \\
& \alpha\left(t_{0}=-5.3 a\right)=(15 \pm 8) \cdot 10^{-4} \mathrm{fm}^{3}
\end{aligned}
$$

which, particularly in the latter case, is in quite good agreement 18 with the results of $[5,7]$. Thus, at the level of the raw numerical measurement, the present work in fact corroborates the results obtained in $[5,7]$; at the same time, it is now clear that such a measurement at a single fixed $t_{0}$ in general contains two separate effects, i.e.,

\footnotetext{
${ }^{16}$ The studies reported in $[5,7]$ employ the quenched approximation.

${ }^{17}$ These estimates were obtained by linearly interpolating results quoted in [7] in $m_{\pi}$.

${ }^{18}$ It should be noted that these comparisons depend sensitively on the determinations of the lattice spacings in the different calculations.
} 
the electric polarizability itself and the effect of subjecting the neutron to a constant external field. To disentangle the former from the latter using such measurements, it is necessary to combine the data obtained using a variety of $t_{0}$. This suggests that it would be worthwhile to supplement the measurements already performed in $[5,7]$ by further analogous measurements at other values of $t_{0}$ in order to obtain a more comprehensive picture of the physical effects engendered by the introduction of the external field and thus be able to isolate the different effects from one another.

\section{$5 \quad$ Summary and outlook}

The investigation reported here represents a first exploration of the neutron electric polarizability in the context of lattice QCD with dynamical quarks. Its main thrust lay in clarifying conceptual questions within the framework of the background field method and assessing the feasibility of numerical computations on that basis, using a $S U(3)$ flavor-symmetric ensemble as a test case.

Two central issues needed to be addressed to arrive at a cogent calculational scheme. On the one hand, the presence of dynamical quarks dictates the use of four-point function methods, introducing, in particular, the need to evaluate disconnected diagrams. These contributions, which were included in the numerical calculations carried out in this work via stochastic estimation, significantly increase the computational expense of the measurement. Nevertheless, the feasibility of carrying out such measurements was demonstrated for the $S U(3)$ flavor-symmetric case; the cost of progress towards lighter quark masses does not seem prohibitive, but such an endeavor will require a, by current standards, significant commitment of computational resources.

On the other hand, a strong emphasis was placed within the present work on the physical consequences of shifting the external electromagnetic field by a constant on a finite lattice. While such shifts merely correspond to gauge transformations in infinite space, on a finite lattice, they influence the physical spectrum and thus mask the mass shift due to the electric polarizability itself. On lattices of a practical size, this effect has a dominating influence on the neutron mass shift, from which one aims to extract the electrical polarizability. To disentangle the two effects, measurements using a variety of external fields which are shifted with respect to one another are necessary (further impacting computational cost). It should be noted that this issue also affects investigations carried out in the quenched approximation, such as reported in $[5,7]$. The present investigation, complementing that effort, suggests additional measurements to supplement the ones already carried out, in order to gain a comprehensive picture of the effects playing a role. It is hoped that the results obtained here will provide motivation and useful input for an expanded measurement program in this direction.

Looking forward, besides the obvious need to progress towards lighter quark masses, it would be interesting to study other hadrons, especially with a view towards measuring 
polarizability combinations in which disconnected diagrams at least partially cancel. Such combinations could be calculated with higher accuracy at lower cost. However, a potential obstacle to this which should be kept in mind is the following: Typically, hadrons of differing electric charge would be involved, and, a priori, it is not clear that measurements using the same external electromagnetic field are appropriate in each case for the purpose of isolating the electric polarizability. On the other hand, results obtained in different external fields cannot be combined straightforwardly to cancel disconnected contributions.

One possibility of avoiding such difficulties lies in using alternate methods of accessing polarizabilities, e.g., via density-density correlation functions [30]. That approach would circumvent the necessity of explicitly introducing an external electromagnetic field. Density-density correlation functions at unequal times can be used to extract hadron polarizabilities, specifically by measuring the second moment (with respect to spatial separation) of the correlation function for a range of relative times and integrating over the latter. When calculating polarizabilities of hadrons in this manner, at least partial cancellations of disconnected diagrams can be achieved straightforwardly by forming the proper isovector combinations. Care must be taken to restrict the hadron momentum to the nonrelativistic regime, in order to exclude relativistic effects which complicate the interpretation of the density-density correlation function and the extraction of the polarizability. Also, density-density correlation functions generally fall off less rapidly than standard hadron wave functions (a doubling of the extent being typical); this has motivated the development of periodic image correction methods [30] which are expected to prove helpful in this context.

\section{Acknowledgments}

The author is especially grateful to J. Negele, K. Orginos and D. Renner for sharing their expertise in numerous discussions. This investigation furthermore benefited from helpful exchanges with R. Brower, M. Burkardt, W. Detmold, R. Edwards, H. Grießhammer, J. Osborn, D. Toussaint and W. Wilcox, as well as from enlightening comments provided by D. B. Kaplan and M. Savage. Also, this work would not have been possible without the dynamical quark configurations made available by members of the MILC Collaboration. It is a pleasure to acknowledge the use of computer resources provided by the U.S. DOE through the USQCD project at Jefferson Lab, and support by the U.S. DOE under grant number DE-FG03-95ER40965.

Note added: The author is grateful to D. Toussaint for pointing out ref. [31], which in an appendix also discusses the electric polarizability of the neutron, and for further exchanges thereon. One particular point emphasized in [31] is that, to properly represent the effect of a classical external electric field, the electric field as introduced in this work 
and also in $[2,5,7]$ should be analytically continued to imaginary values. This further step, which was not carried out in $[2,5,7]$ and in the present treatment, implies that the results (58) and (59) receive an additional overall minus sign. In view of the small magnitude of (58) and (59), this does not decisively impact the further conclusions drawn in the present work. However, it will need to be taken into account, and revisited in more detail, in work going forward aiming at progress towards lighter pion masses.

\section{References}

[1] D. Babusci, G. Giordano, A. I. L'vov, G. Matone and A. M. Nathan, Phys. Rev. C 58 (1998) 1013.

[2] H. R. Fiebig, W. Wilcox and R. M. Woloshyn, Nucl. Phys. B324 (1989) 47.

[3] W. Wilcox, Ann. Phys. 255 (1997) 60.

[4] W. Wilcox, Phys. Rev. D 57 (1998) 6731.

[5] J. Christensen, F. X. Lee, W. Wilcox and L. Zhou, Nucl. Phys. Proc. Suppl. 119 (2003) 269.

[6] L. Zhou, F. X. Lee, W. Wilcox and J. Christensen, Nucl. Phys. Proc. Suppl. 119 (2003) 272.

[7] J. Christensen, W. Wilcox, F. X. Lee and L. Zhou, Phys. Rev. D 72 (2005) 034503.

[8] F. X. Lee, L. Zhou, W. Wilcox and J. Christensen, Phys. Rev. D 73 (2006) 034503.

[9] F. X. Lee, L. Zhou, W. Wilcox and J. Christensen, PoS LAT2005 (2006) 031.

[10] W. Detmold, B. C. Tiburzi and A. Walker-Loud, Phys. Rev. D 73 (2006) 114505.

[11] K. Orginos and D. Toussaint (MILC Collaboration), Phys. Rev. D 59 (1998) 014501.

[12] K. Orginos, D. Toussaint, and R. L. Sugar (MILC Collaboration), Phys. Rev. D 60 (1999) 054503.

[13] C. W. Bernard, T. Burch, K. Orginos, D. Toussaint, T. A. DeGrand, C. DeTar, S. Datta, S. A. Gottlieb, U. M. Heller and R. Sugar, Phys. Rev. D 64 (2001) 054506 .

[14] C. Aubin, C. Bernard, C. DeTar, S. A. Gottlieb, E. B. Gregory, U. M. Heller, J. E. Hetrick, J. Osborn, R. Sugar and D. Toussaint, Phys. Rev. D 70 (2004) 094505 .

[15] A. Hasenfratz and F. Knechtli, Phys. Rev. D 64 (2001) 034504.

[16] R. G. Edwards, G. T. Fleming, P. Hägler, J. W. Negele, K. Orginos, A. Pochinsky, D. B. Renner, D. G. Richards and W. Schroers (LHPC Collaboration), Phys. Rev. Lett. 96 (2006) 052001. 
[17] P. Hägler, W. Schroers, J. Bratt, R. G. Edwards, M. Engelhardt, G. T. Fleming, B. Musch, J. W. Negele, K. Orginos, A. V. Pochinsky, D. B. Renner and D. G. Richards (LHPC Collaboration), arXiv:0705.4295.

[18] D. B. Kaplan, Phys. Lett. B288 (1992) 342.

[19] Y. Shamir, Nucl. Phys. B406 (1993) 90.

[20] C. Alexandrou, F. Jegerlehner, S. Güsken, K. Schilling and R. Sommer, Phys. Lett. B256 (1991) 60.

[21] D. Dolgov, R. Brower, S. Capitani, P. Dreher, J. W. Negele, A. Pochinsky, D. B. Renner, N. Eicker, T. Lippert, K. Schilling, R. G. Edwards and U. M. Heller (LHPC and SESAM Collaborations), Phys. Rev. D 66 (2002) 034506.

[22] R. G. Edwards and B. Joó (LHPC and UKQCD Collaborations), Nucl. Phys. Proc. Suppl. 140 (2005) 832.

[23] C. McClendon, JLab preprint JLAB-THY-01-29.

[24] A. Ó Cais, K. Juge, M. Peardon, S. Ryan and J.-I. Skullerud (TrinLat Collaboration), Nucl. Phys. Proc. Suppl. 140 (2005) 844.

[25] W.-M. Yao et al., J. Phys. G: Nucl. Part. Phys. 33 (2006) 1.

[26] V. Bernard, N. Kaiser, A. Schmidt and U.-G. Meißner, Phys. Lett. B319 (1993) 269.

[27] T. R. Hemmert, B. R. Holstein and J. Kambor, Phys. Rev. D 55 (1997) 5598.

[28] R. P. Hildebrandt, H. W. Grießhammer, T. R. Hemmert and B. Pasquini, Eur. Phys. J. A 20 (2004) 293.

[29] S. R. Beane, M. Malheiro, J. A. McGovern, D. R. Phillips and U. van Kolck, Phys. Lett. B567 (2003) 200; erratum ibid. B607 (2005) 320.

[30] M. Burkardt, J. M. Grandy and J. W. Negele, Ann. Phys. 238 (1995) 441.

[31] E. Shintani, S. Aoki, N. Ishizuka, K. Kanaya, Y. Kikukawa, Y. Kuramashi, M. Okawa, A. Ukawa and T. Yoshie, Phys. Rev. D 75 (2007) 034507. 